\title{
A Review of Carbon-Based Materials for Safe Lithium Metal Anodes
}

\author{
Yan Liu ${ }^{1,2}$, Xifei $\mathrm{Li}^{1,2,3 *}$, Linlin Fan ${ }^{1,2}$, Shufeng $\mathrm{Li}^{1 *}$, Hirbod Maleki Kheimeh Sari ${ }^{1,2}$ and \\ Jian Qin $^{1,2}$
}

${ }^{1}$ School of Materials Science and Engineering, Institute of Advanced Electrochemical Energy, Xi'an University of Technology, Xi'an, China, ${ }^{2}$ Shaanxi International Joint Research Center of Surface Technology for Energy Storage Materials, Xi'an, China, ${ }^{3}$ State Center for International Cooperation on Designer Low-Carbon \& Environmental Materials (CDLCEM), Zhengzhou

University, Zhengzhou, China

OPEN ACCESS

Edited by:

Wenping Sun,

University of Wollongong, Australia

Reviewed by:

Jiantie Xu,

South China University of

Technology, China

Yinzhu Jiang,

Zhejiang University, China

*Correspondence:

Xifei $\mathrm{Li}$

xfli2011@hotmail.com

Shufeng Li

shufengli@xaut.edu.cn

Specialty section:

This article was submitted to

Electrochemistry,

a section of the journal

Frontiers in Chemistry

Received: 07 August 2019

Accepted: 10 October 2019

Published: 04 November 2019

Citation:

Liu Y, Li X, Fan L, Li S, Maleki Kheimeh Sari H and Qin J (2019) A Review of Carbon-Based Materials for Safe Lithium Metal Anodes.

Front. Chem. 7:721.

doi: 10.3389/fchem.2019.00721
Lithium metal is a promising anode material with extremely high theoretical specific capacity $\left(3,860 \mathrm{~mA} \mathrm{~h} \mathrm{~g} \mathrm{~g}^{-1}\right)$, low density $\left(0.59 \mathrm{~g} \mathrm{~cm}^{-3}\right)$, and the lowest negative electrochemical potential of all potential candidates $(-3.04 \mathrm{~V} v \mathrm{vs}$. the standard hydrogen electrode). However, uncontrollable Li dendrite growth leads to a short lifespan and catastrophic safety hazards, which has restricted its practical application for many years. Some effective strategies have been adopted regarding these challenges, including electrolyte modification, introducing a protective layer, nanostructured anodes, and membrane modification. Carbon-based materials have been demonstrated to significantly address the challenge of Li dendrites. In this review, carbon-based materials and their application and challenges in lithium metal anode protection have been discussed in detail. In addition, the applications of lithium anodes protected by carbon-based materials in Li-S batteries and $\mathrm{Li}^{-} \mathrm{O}_{2}$ batteries have been summarized.

Keywords: lithium metal anodes, dendrite, electrochemical performance, carbon-based materials, energy storage

\section{INTRODUCTION}

Environmental pollution has become a serious issue, and green energies, including batteries, particularly rechargeable lithium-metal batteries, have received extensive attention from researchers. Lithium metal is the ultimate choice for the anode in lithium batteries due to it having the highest theoretical capacity $\left(3,860 \mathrm{~mA} \mathrm{~h} \mathrm{~g}^{-1}\right.$ or $\left.2,061 \mathrm{~mA} \mathrm{~h} \mathrm{~cm}^{-3}\right)$ and lowest electrochemical potential ( $-3.04 \mathrm{~V}$ vs. the standard hydrogen electrode) (Tarascon and Armand, 2001; Wu et al., 2014) among all the possible candidates (Lin et al., 2017).

However, growth of Li dendrites during repeated charge/discharge processes and low Coulombic efficiency (CE) (Wu et al., 2014; Maleki Kheimeh Sari and Li, 2019) lead to early cell death, rapid cycling capacity decay, and catastrophic thermal runaway (Yingying et al., 2014; Zheng et al., 2014; Kai Z. et al., 2016; Zhang R. et al., 2016), which substantially impede the development of lithium metal batteries (LMBs). Hence, although the theoretical capacity of a lithium metal anode is about ten times greater than that of a graphite anode $\left(372 \mathrm{~mA} \mathrm{~h} \mathrm{~g}^{-1}\right)$, it has not yet been commercialized. Furthermore, compared to lithium-ion batteries (LIBs, specific energy $\sim 250 \mathrm{Wh} \mathrm{kg}^{-1}$ ), Li-S and $\mathrm{Li}-\mathrm{O}_{2}$ systems can further boost specific energies to up to $\sim 650$ and $950 \mathrm{Wh} \mathrm{kg}^{-1}$ (Lin et al., 2017), respectively. Therefore, it can be speculated that in the near future lithium metal anodes will be an indispensable part of $\mathrm{Li}-\mathrm{S}, \mathrm{Li}-\mathrm{O}_{2}$, and $\mathrm{Li}-\mathrm{CO}_{2}$ batteries, realizing a high specific capacity to fulfill the ever-growing energy density requirements of portable electronic devices and electrical vehicles (Bruce et al., 2011; Aurbach et al., 2016; Zhi et al., 2016; Lim et al., 2017). The only important barrier to this development is the growth of lithium dendrites, which needs to be curtailed. 
Certain methods have been adopted in this regard, including using $\mathrm{LiX}$ alloys $(\mathrm{X}=\mathrm{Al}, \mathrm{B}, \mathrm{Si}, \mathrm{Sn}, \mathrm{C}$, etc.), organic electrolyte and Li metal/electrolyte interface modifications, solidstate electrolytes, and structured anode designs (Cheng et al., 2015), leading to some satisfactory results. Notably, many researchers have used carbon-based materials to protect lithium metal anodes and have made significant progress due to their advantageous characteristics, including superior thermal and electrical conductivity, high temperature resistance, excellent chemical stability, good mechanical strength, and satisfactory lubrication performance.

In this review, we first elaborate on the formation mechanism of lithium dendrites and then summarize the application of carbon-based materials and their composites in lithium metal anode protection. The targeted carbon-based materials include $\mathrm{C}_{60}$, carbon nanotubes $(\mathrm{CNT})$, graphene, graphite, and graphdiyne (GDY), which was first synthesized by Li's group in 2010 (Guoxing et al., 2010). The aforementioned graphite-family materials and their composites deliver perfect electrochemical performance, which makes them one of the best options for lithium metal anode protection. The review then goes on to discuss the potential applications and challenges of lithium metal anodes protected by carbon-based materials in $\mathrm{Li}-\mathrm{S}$ and $\mathrm{Li}-\mathrm{O}_{2}$ batteries in general.

\section{THE FORMATION MECHANISM OF LI DENDRITES}

The dendritic growth of Li metal has been investigated since the 1960s, and research on Li metal anodes has continued without break during the past 40 years (Suo et al., 2012; Whittingham, 2012; Hong-Jie et al., 2014; Manthiram et al., 2014; Mukherjee et al., 2014; Cheng and Zhang, 2015; Jiulin et al., 2015; Peng et al., 2015). The formation process of lithium dendrites and dead lithium and the related safety risks and lifespan impacts are shown in Figure 1. Although many studies have been devoted to exploring the growth mechanism of these dendrites, its exact nature is still inconclusive. Due to the uneven surface of the lithium sheet, lithium ions are continuously deposited and stripped through charging and discharging; under the influence of space charge, convex areas of the surfacetend to accumulate lithium ions, eventually leading to inhomogeneous deposition of lithium ions and formation of dendrites. The dendrites will pierce the separator, resulting in a short circuit of the cell, which is a potential security issue and can cause a serious accident. Therefore, it is important to understand the formation mechanism of $\mathrm{Li}$ dendrites and to find a novel method for restraining it.

There is a widely accepted diffusion model (Equation 1) called the "Sand's time $\tau$," which is applied to describe the migration properties of lithium ions and electrons (Chazalviel, 1990; Fleury et al., 1990; Brissot et al., 1998; Rosso et al., 2001). Sand's time was proposed for the first time when, Sand (1901) investigated the liberation of hydrogen in a mixture of copper sulfate and sulfuric acid, and it has since been used more generally, including for LMBs.
In the LMB system, the time at which lithium dendrites begin to grow is called "Sand's time."

$$
\tau=\pi D\left(\frac{e C_{0}}{2 J t_{a}}\right)^{2}
$$

In Equation (1), $\mathrm{D}$ is the ambipolar diffusion coefficient, $D=$ $\left(\mu_{\mathrm{a}} D_{\mathrm{c}}+\mu_{\mathrm{c}} D_{\mathrm{a}}\right) /\left(\mu_{\mathrm{a}}+\mu_{\mathrm{c}}\right)$, where $D_{\mathrm{c}}$ and $D_{\mathrm{a}}$ are cationic and anionic diffusion coefficients, $e$ is the electronic charge, $C_{0}$ is the initial concentration of Li salt, $\mu_{\mathrm{a}}$ and $\mu_{\mathrm{c}}$ are anionic and cationic mobilities, respectively, $J$ is the effective electrode current density, and $t_{\mathrm{a}}$ is the anionic transport number $t_{\mathrm{a}}=\mu_{\mathrm{a}} /\left(\mu_{\mathrm{a}}+\mu_{\mathrm{c}}\right)$.

In the "Sand's time" model, $J$ and $t_{a}$ are in inverse proportion to $\tau$. If $J$ or $t_{a}$ decreases and the other parameters remain constant, Sand's time $(\tau)$ gets larger, which indicates that the cell has a long lifespan before the growth of Li dendrites (Zhang R. et al., 2016). Equation (2) can predict two different behaviors for a symmetrical cell (Brissot et al., 1999). One is when $\delta \mathrm{C} / \delta \mathrm{x}<$ $2 \mathrm{C}_{0} / \mathrm{L}$ (Figure 2A), in which case the ionic concentration profile evolves to a steady state at which the concentration gradient is constant (Bruce and Vincent, 1987) and the voltage is also stable. The other is when $\delta \mathrm{C} / \delta \mathrm{x}>2 \mathrm{C}_{0} / \mathrm{L}$ (Figure 2B), in which case the concentration will evolve to reach zero at the negative electrode at Sand's time (Brissot et al., 1999). Meanwhile, the voltage becomes very unstable until it eventually drops to zero at Sand's time due to the ionic concentrations in the vicinity of the negative electrode (Sand, 1901). If the current density $(J)$ is greater than the critical value $\left(J^{*}\right)$, the aforementioned phenomenon will appear; Equation (3) represents the critical value $J^{*}$. The concentration of cations and anions will then endure a dramatic change, resulting in the aggregation of a large amount of positive charge in the negative electrode, which leads to a local space charge (Brissot et al., 1999).

$$
\begin{gathered}
\frac{\partial C}{\partial x}(x=0)=\frac{-J}{e D\left(1+\frac{\mu_{a}}{\mu_{c}}\right)} \\
J^{*}=\frac{2 e C_{0} D}{t_{a} L}
\end{gathered}
$$

The theory of space charge was put forward by Fleury et al. (1990) and can explain the growth process of lithium dendrites. A thin rectangular cell model was constructed by Chazalviel in a dilute solution under a strong electric field (Figure 2C). Chazalviel found that diffusion and migration play a dominant role in different regions of the rectangular cell and calculated the distributions of potential and of ion concentration (Figure 2E). Region $\mathrm{I}\left(\mathrm{Z}_{\mathrm{c}} \mathrm{C}_{\mathrm{c}} \approx \mathrm{Z}_{\mathrm{a}} \mathrm{C}_{\mathrm{c}}\right)$ is called the quasi-neutral region, where the electrostatic potential is close to zero and $\mathrm{V}_{0}$ at $\mathrm{x}=0$ and $\mathrm{x}=$ $\mathrm{L}$, respectively. The region extends from the anode to the vicinity of the cathode and dominates most areas of the cell, controlled by a diffusion mechanism. Region II $\left(\mathrm{C}_{\mathrm{a}}<<\mathrm{C}_{\mathrm{c}}\right)$ is called the space-charge region, which is near the cathode, occupies a very small fraction of the cell, and is controlled by the migration of the electric field. Indeed, the electron transfer speed is faster than the 


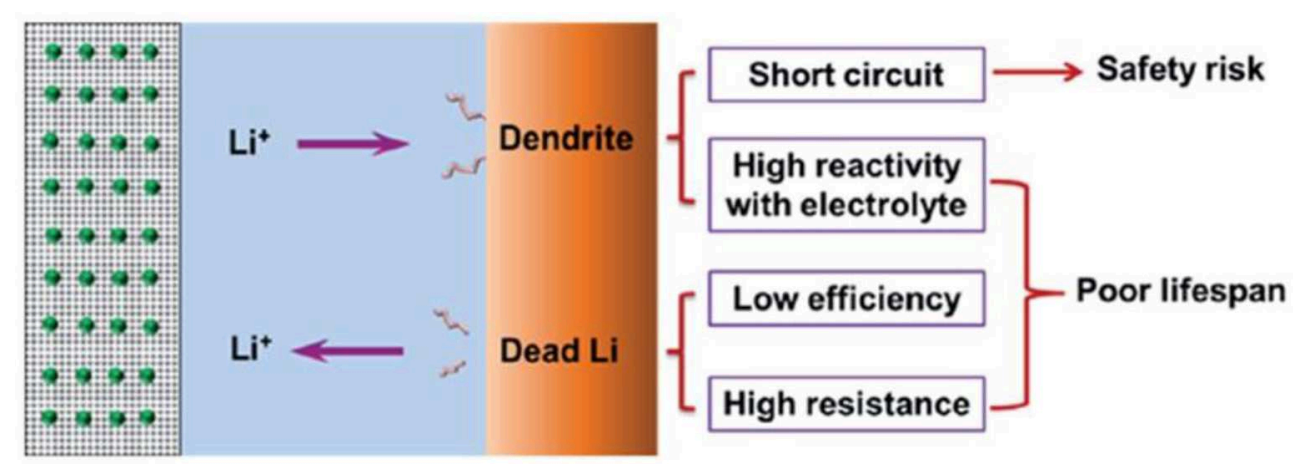

FIGURE 1 | Schematic diagram of the typical morphology of Li dendrites and the related safety risks and lifespan effects. Reprinted with permission from Cheng et al. (2015). Copyright (2015) Royal Society of Chemistry.

ion diffusion rate. The ion concentration $\left(\mathrm{C}_{\mathrm{a}}, \mathrm{C}_{\mathrm{b}}\right)$ and potential (V) change over time (Figure 2D). The electric field is distributed evenly, and no space charge is generated at $t=0$. The positive ions migrate from anode to cathode, resulting in congregation of negative ions near the anode. Depletion develops in the vicinity of the cathode, making it a space-charge region. However, the congregation of anions in the anode leads to enhancement of the local electric field and the attraction of more cations, and the intensified electric field results in rapid cation deposition. In fact, lithium ions are being constantly released and embedded during the charging and discharging process, and the charge on the protruding part of the lithium metal surface tends to be inhomogeneously distributed, which generates a space-charge region and consequently dendrites. Hence, dendrite growth can be inhibited by controlling the formation of a space-charge region. This can be done by increasing the convective motions of the electrolyte, which can effectively control the stirring rate, salt solution concentration gradient, and current density.

\section{CARBON-BASED MATERIALS FOR SAFE LITHIUM METAL ANODES}

Many effective methods have been adopted thus far to inhibit lithium dendrite growth. Among them, researchers have carried out a lot of work using carbon-based materials to protect lithium metal anodes and have made great progress. The interfacial energy between carbon-based materials and Li metal is vital to this process. $\mathrm{Li}$ is body-centered cubic (BCC) and belongs to the $\operatorname{Im} 3 \mathrm{~m}$ space group, while $\mathrm{C}$ is categorized into the cubic system (diamond) or hexagonal system (graphite) and belongs to the $\mathrm{Fd} 3 \mathrm{~m}$ (diamond) space group and $\mathrm{P} 6 / \mathrm{mmm}$ (graphite) space group. This mismatch between the two-phase crystal structure increases because of the interface energy. Additionally, their radii are different: the radius of $\mathrm{C}$ is $0.077 \mathrm{~nm}$, while that of $\mathrm{Li}$ is nearly double that (i.e., $0.152 \mathrm{~nm}$ ). From the phase diagram of lithium and carbon (Figure 3A), it is evident that lithium and carbon can form an alloy phase while overpotential (about $14 \mathrm{mV}$ ) still exists (see Figure 3B); indeed, carbon materials have no solubility in lithium. Moreover, compared with $\mathrm{Cu}$ and $\mathrm{Ni}$, the voltage dip of carbon is relatively small, which means that the nucleation overpotential [i.e., the difference between the bottom of the voltage drop and the flat portion of the voltage platform (Kai Y. et al., 2016)] of Li metal is much smaller. In this section, the application of different types of carbon materials, carbon materials modified by doping, and composites with carbon materials in lithium protection are thoroughly reviewed.

\section{Types of Carbon Materials for Lithium Metal Anode Protection}

Based on their morphologies, carbon materials can be categorized into several groups including fullerenes $\left(\mathrm{C}_{60}\right)$, graphite, $\mathrm{CNT}$, graphene, and graphdiyne (GDY). It is well-known that different morphologies can show different physicochemical performances. In this section, the application of carbon materials of different types for lithium metal protection is introduced.

\section{Fullerenes $\left(\mathrm{C}_{60}\right)$}

In 1985, fullerenes were firstly discovered by scientists at Ross University in Texas. There are $60 \mathrm{C}$ atoms in a $\mathrm{C}_{60}$ molecule, which form 32 planes, 20 regular hexagons, and 12 regular pentagons. The fullerene structure is similar to that of graphite. Graphite is a graphene layer composed of six-membered rings, whereas fullerene contains five-, six-, and seven-membered rings. In addition, the carbon atoms in fullerenes are bonded together in a spherical dome structure. It can be classified among molecular crystals with a low melting point, low hardness, and insulative properties. If they suffer from high-intensity photon or electron irradiation or interact with plasma (Arie et al., 2008), fullerenes can evolve from the ground state and form polymeric materials. Indeed, chemically modified synthetic fullerenes have been used by some researchers in the field of electrochemistry (Giacalone and Nazario, 2006). Interestingly, Arie et al. (2008) adopted three methods (i.e., radio frequency magnetron sputtering, plasma, and ion-assisted thermal evaporation) to coat a Li metal surface with fullerenes. The results show that the $\mathrm{C}_{60}$ film formed through ion-assisted thermal evaporation is the thickest (Figure 3C), that from plasma-assisted evaporation is moderate (Figure 3D), and that from magnetron sputtering is the finest (Figure 3E). As observed in Figure 3F, coating the surface of lithium metal 
A

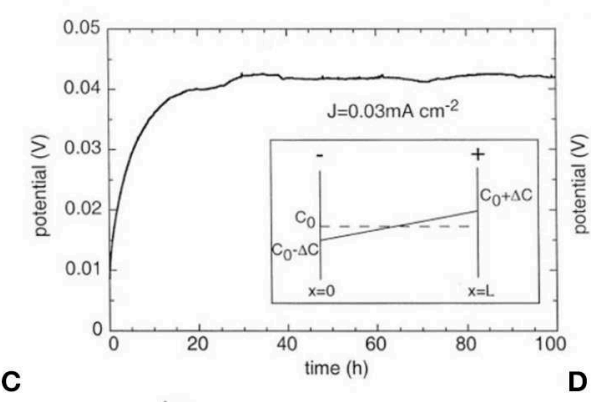

C

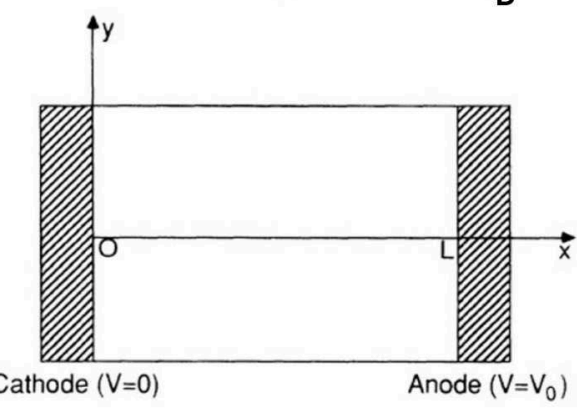

B
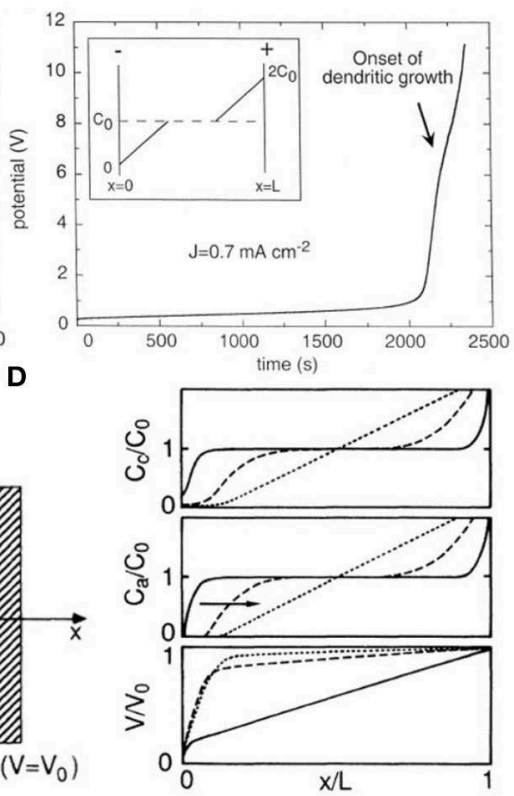

E
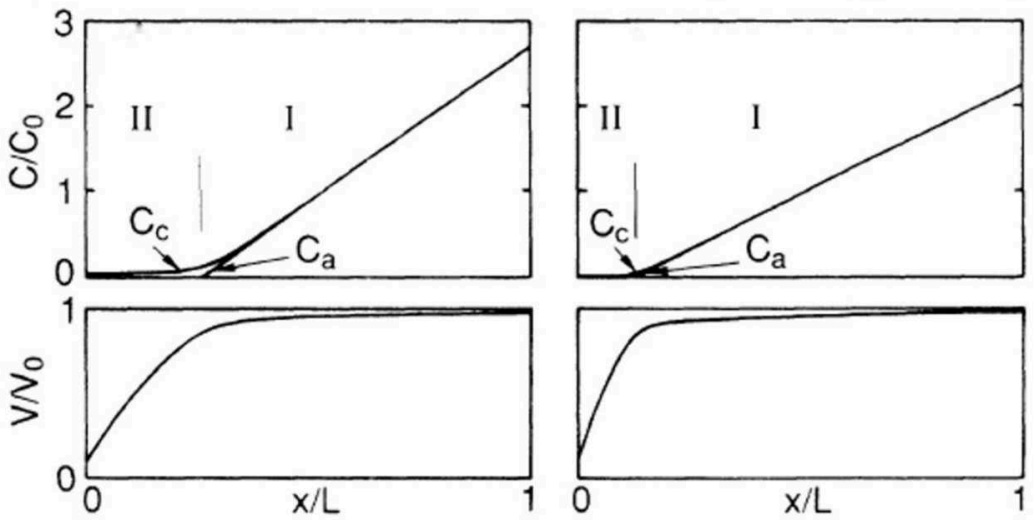

FIGURE 2 | For a given distance $L$ between the electrodes, (A) if $J<J^{*}$, the system evolves to a steady state where the concentration varies linearly from $\mathrm{C}_{0}$ to $\Delta C$ at the negative electrode, (B) if $J<J^{*}$ (semi-infinite approximation), the ionic concentration drops to zero and the cell potential diverges at Sand's time. Here, Sand's time is about 2,100 s [note the different time scales in $(\mathbf{A}, \mathbf{B})$ ]. The inflection on the $V(t)$ curve shown by the arrow corresponds to the onset of dendritic growth. Reprinted with permission from Brissot et al. (1999). Copyright (1999) Elsevier Science BV. (C) Schematic diagram of the cell. (D) Profile of the ion concentrations and electrostatic potentials as a function of time. (E) Profile of the ion concentrations $\mathrm{C}_{\mathrm{c}}$ and $\mathrm{C}_{\mathrm{a}}$ and the electrostatic potential $\mathrm{V}$ resulting from the numerical simulation in the hypothetical case of uniform deposition with negligible growth of the cathode $\left(\mathrm{C}_{0}=10^{10} \mathrm{~cm}^{-3}\right)$. (D) Profile of the ion concentrations $\mathrm{C}_{c}$ and $\mathrm{C}_{a}$ and the electrostatic potential $\vee$ resulting from the numerical simulation in the hypothetical case of uniform deposition with negligible growth of the cathode $\left(\mathrm{C}_{0}=10^{11} \mathrm{~cm}^{-3}\right)$. Reprinted with permission from Chazalviel (1990). Copyright (1990) American Physical Society.

with a carbon film can yield better cycle stability compared to pure lithium metal. It can be seen from Figure 3G that the absorbance plot of the carbon film resulting from the ionbeam assisted technique shows a similarity to that of pure $\mathrm{C}_{60}$. Both of them show three peaks in the 200 to $400 \mathrm{~nm}$ wavelength range. The spectrum provided by the ion beam case shows smaller peaks in comparison to that of pure $\mathrm{C}_{60}$, indicating that this carbon film may contain a portion of initial fullerene $\mathrm{C}_{60}$. Thus, the $\mathrm{C}_{60}$-coated lithium electrode obtained by ion-assisted thermal evaporation displayed the best electrochemical performance.

\section{Carbon Nanotubes (CNTs)}

Carbon nanotubes possess good mechanical properties, good adhesion, and excellent electrical conductivity, thereby improving the interface properties (Wang et al., 2013). Moreover, because of their large internal space and specific surface area, CNTs can alleviate the volume expansion of LMBs during continuous deposition/dissolution. Meanwhile, the binding energy between lithium and CNT is $1.19 \mathrm{eV}$ (Figure 4A), which is lower than that with copper $(2.57 \mathrm{eV})$, graphene $(3.64 \mathrm{eV})$, and $\mathrm{N}(4 \mathrm{eV})$ (Zhang H. et al., 2018). CNTs have smaller RSEI than the pristine lithium, resulting from the better wettability of 
A

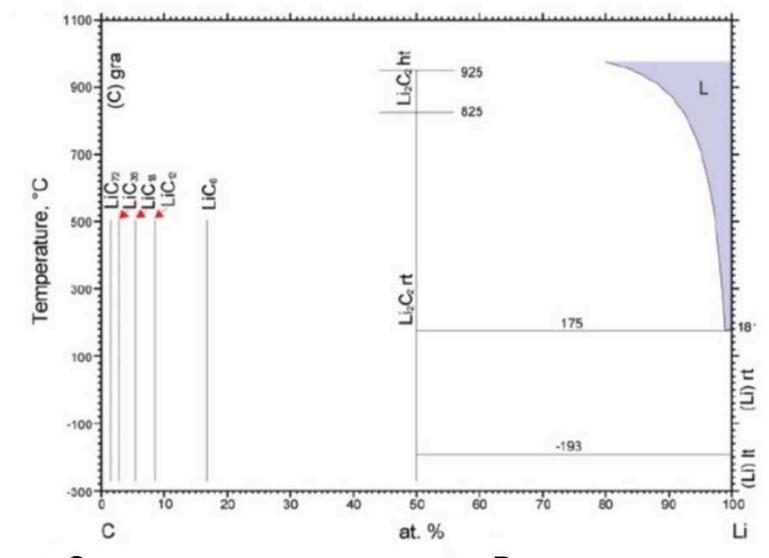

C

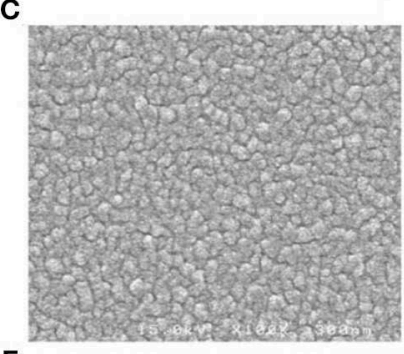

$\mathbf{F}$

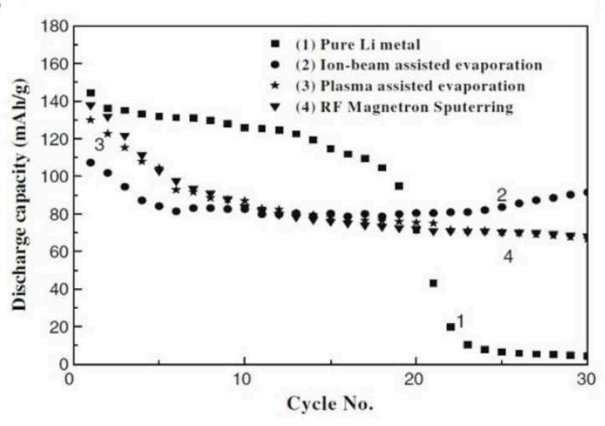

B

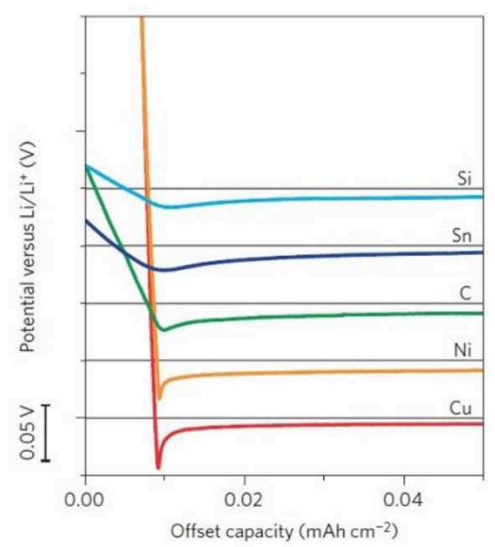

E
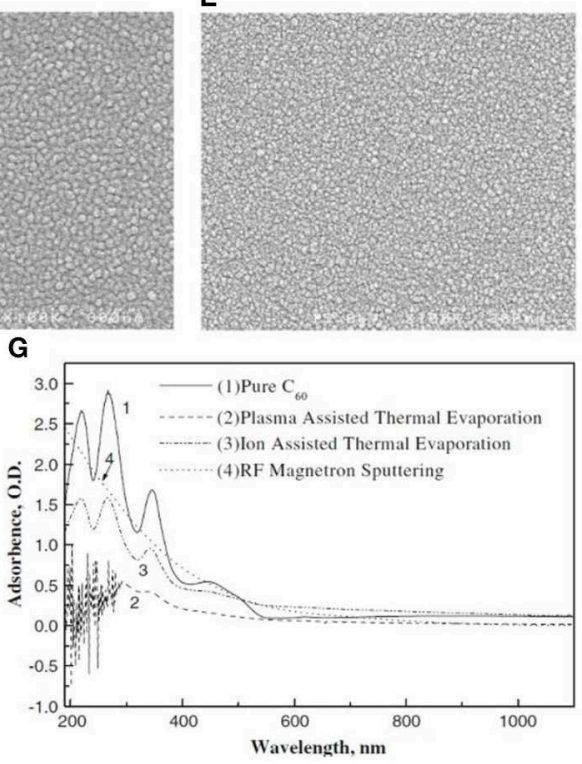

FIGURE 3 | (A) The phase diagram of lithium and carbon. (B) Shifted voltage profiles of various materials with negligible solubility in Li during Li deposition at a current density of $10 \mu \mathrm{A} \mathrm{cm}^{-2}$. Reprinted with permission from Kai Y. et al. (2016). Copyright (2016b) Springer Nature Limited. SEM images of fullerene $\mathrm{C}_{60}$ film deposited by three different techniques (C) Ion beam assisted evaporation. (D) Plasma assisted evaporation and (E) radio frequency magnetron sputtering. (F) The cyclic performance of a pure lithium electrode and fullerene $\mathrm{C}_{60}$-coated lithium electrodes obtained by three different techniques. (G) UV-Vis spectra of the pure $\mathrm{C}_{60}$ film and $\mathrm{C}_{60}$ coated films obtained by three vacuum techniques. Reprinted with permission from Arie et al. (2008). Copyright (2008) Springer Nature Limited.

the interface between the electrode surface and the electrolyte. As shown in Figures 4C,D, in the Zhang D. et al. (2016) study, the resistance of the battery with a CNT buffer layer was $50 \Omega$ before the test, while the resistance of the battery without a CNT buffer layer was $320 \Omega$. It is obvious that the interfacial resistance of the battery with the buffer layer was smaller and more stable, which indicates higher interfacial stability and better electrode-electrolyte contact. This excellent electrode wettability is advantageous for facilitating diffusion of the electrolyte into the inner space of the electrode, thus reducing the transfer resistance due to the reduced ion diffusion length during charge and discharge (Bai et al., 2018). Therefore, the better wettability of CNTs at the electrode surface and electrolyte interface can be observed through electrochemical impedance spectroscopy (EIS). Thus, CNT is considered to be a promising three-dimensional substrate material for lithium metal anodes.

Nakanishi's group used Ni foil and a CNT matrix as the working electrode and lithium foil as the reference electrode (Matsuda et al., 2017). As shown in Figure 4B, the 1st and 5th cycles at a current density of $1.0 \mathrm{~mA} \mathrm{~cm}{ }^{-2}$ and capacity of $1.0 \mathrm{~mA} \mathrm{~h} \mathrm{~cm}{ }^{-2}$ revealed that the CNT matrix has superior electrochemical stability compared to Ni foil. This is because a more stable solid electrolyte membrane is formed in the CNT matrix during continuous deposition/dissolution. According to Figure 4E, the micrometer-sized Li-metal deposits are evenly distributed on the surface of the $\mathrm{Ni}$ foil, but the magnified 


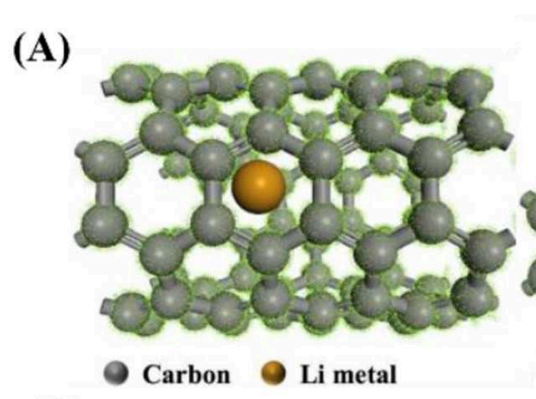

(C)

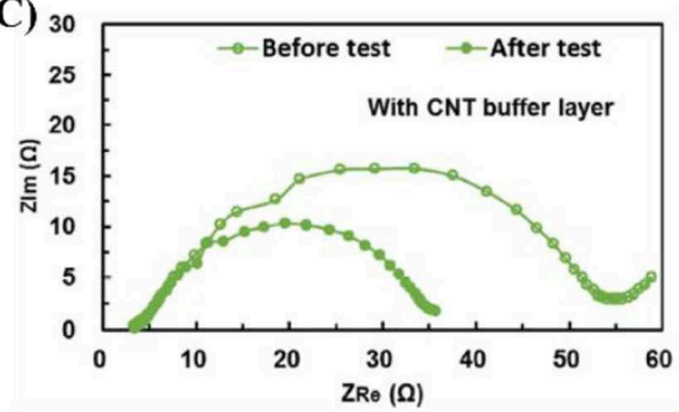

(B) a

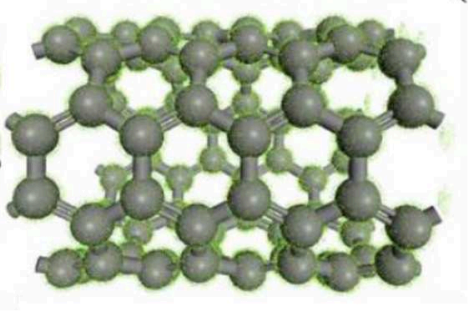

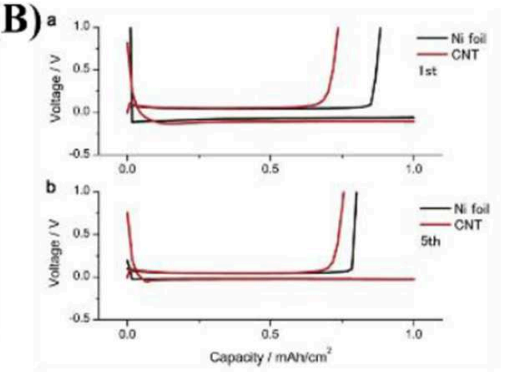

(D) 200

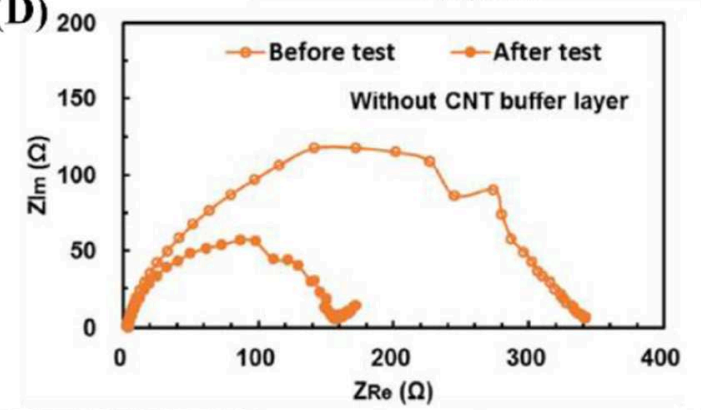

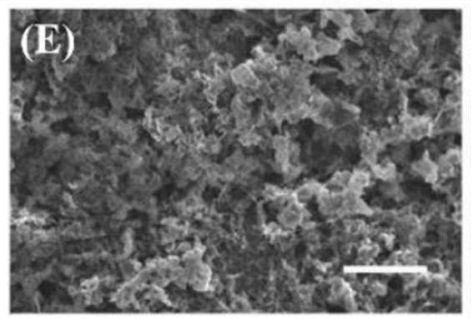
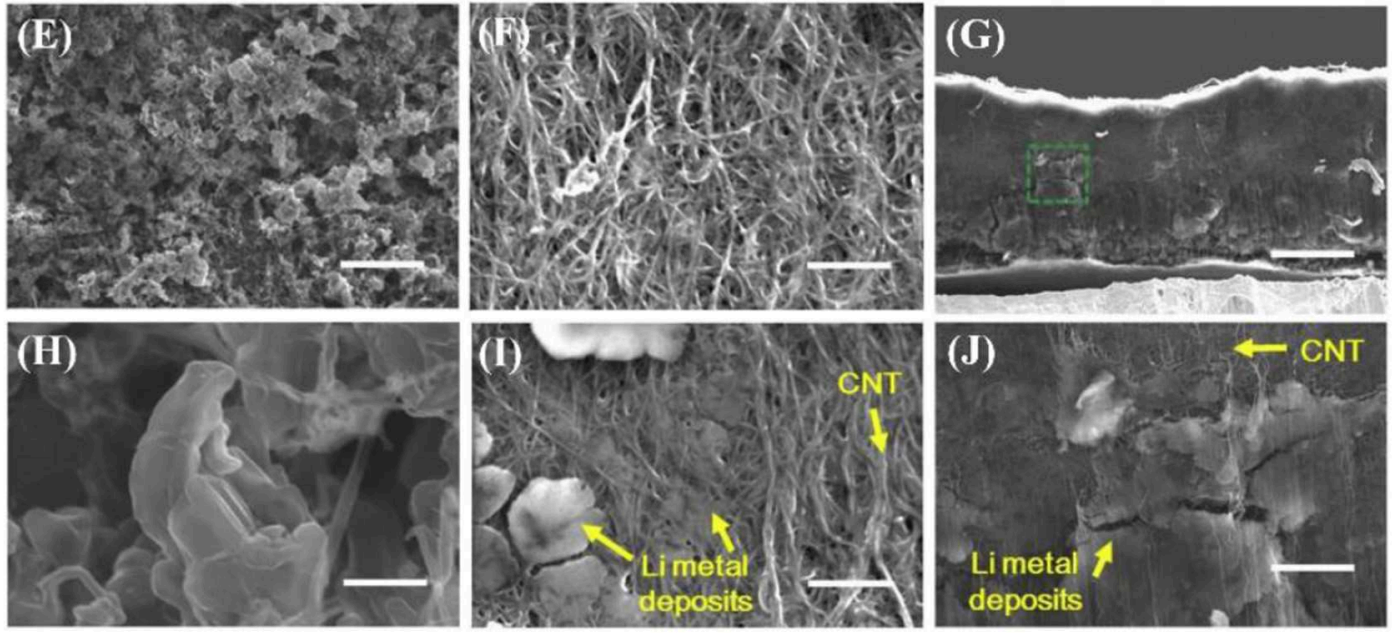

FIGURE 4 | (A) Top view and cross-section view of Li atom binding with carbon atom of CNT calculated with DFT using the CASTEP code in Materials Studio, Accelrys Inc. (version 2017 R2). The local potential of CNT is shown as green dots. Reprinted with permission from Zhang H. et al. (2018). Copyright (2018) Springer Nature Limited. (B) Voltage profiles of electrochemical cells with Ni foil (black curve) or CNT matrix (red curve) as the substrate material during Li-metal deposition/dissolution for the (top) 1 st and (bottom) 5 th cycle at a current density of $1.0 \mathrm{~mA} \mathrm{~cm}^{-2}$ and capacity of $1.0 \mathrm{~mA} \mathrm{~h} \mathrm{~cm}^{-2}$. EIS curves at frequencies ranging from $100 \mathrm{kHz}$ to $100 \mathrm{mHz}$ of the symmetric battery (C) with and (D) without the CNT buffer layer before the test and after 40 charge-discharge cycles. Reprinted with permission from Zhang D. et al. (2016). Copyright (2016b) Royal Society of Chemistry. (E,H) Top-view SEM images of Ni foil taken out from the electrochemical cell after the 5th Li-metal deposition process. (F,I) Top-view and (G,J) side-view SEM images of CNT matrix taken out from the electrochemical cell after the 5th Li-metal deposition process. The scale bar is $20 \mathrm{~mm}$ in $\mathbf{E , G}$, and $2 \mathrm{~mm}$ in $\mathbf{F}, \mathbf{H}-\mathbf{J}$. Reprinted with permission from Matsuda et al. (2017). Copyright (2017) Elsevier Science BV.

SEM shows dendritic growth (Figure $4 \mathbf{H}$ ). In contrast, the surface of the CNTs is smooth, without any dendrite formation (Figure 4G). The lithium deposit is clearly observable in the SEM images in Figure 4I (top-view) and Figures 4G,J (side view).

Combining Li metal with CNTs to form a Li-CNT electrode can properly guide the $\mathrm{Li}^{+}$plating, alleviating the volume expansion and inhibiting dendrite growth (Wang Y. et al., 2017). According to the latter report, not only can the Li-CNT composite electrode accommodate the small-sized Li metal, but it can also maintain the shape of the carbon nanotube frame.
Compared with pure lithium foil, a Li-CNT electrode is beneficial for reducing the probability of lithium dendrite formation and has a superior coulombic efficiency (CE). Many articles have reported that the $\mathrm{CE}$ directly obtained by the electrode during the plating/stripping process is not well used for pre-storing lithium (Ding et al., 2013; Lin et al., 2016; Liu et al., 2016). However, it is undeniable that Li-CNT electrodes can effectively inhibit dendrite growth (Figure 5A). At current densities of 0.1 and $0.5 \mathrm{~mA} \mathrm{~cm}^{-2}$, the overpotential of the pure lithium foil electrode shows a trend from large to small and finally stabilizes, while the 


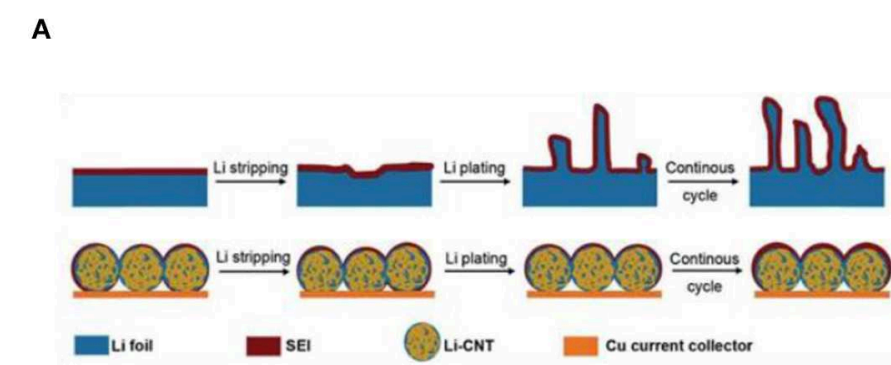

C

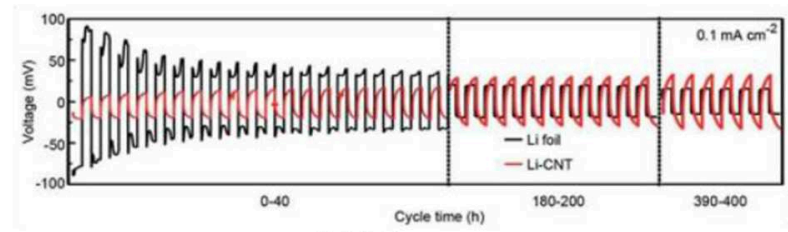

Li foil

D

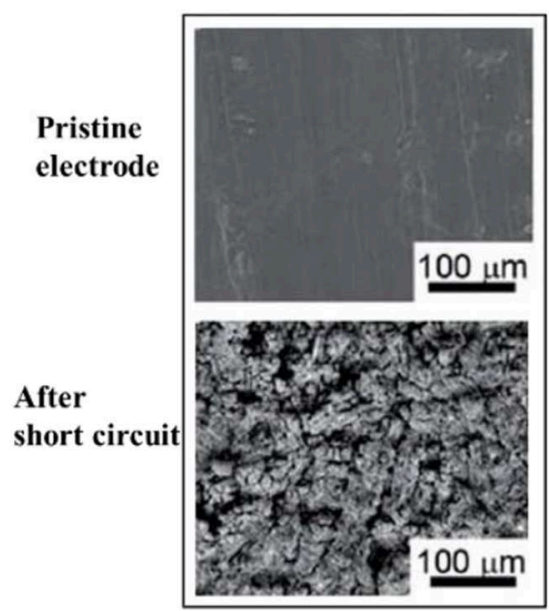

B
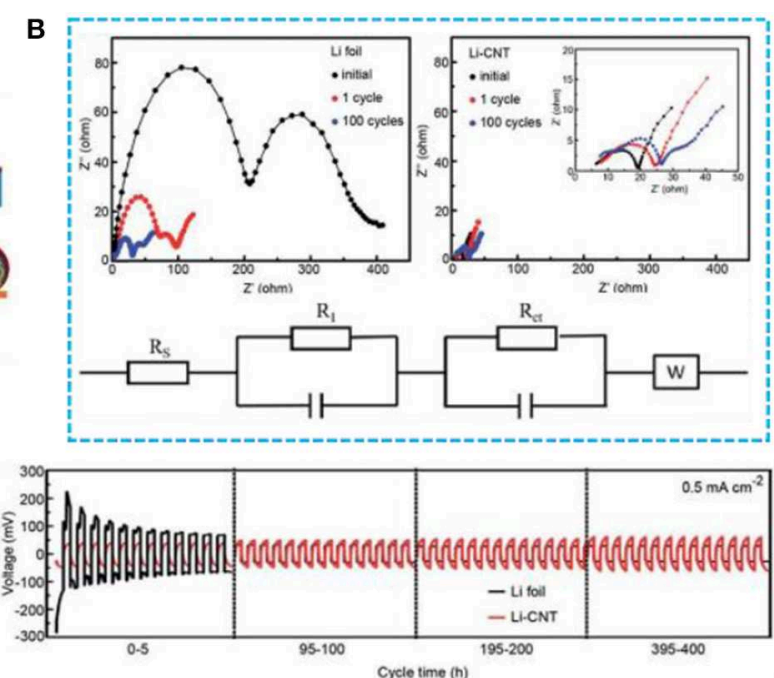

Li-CNT
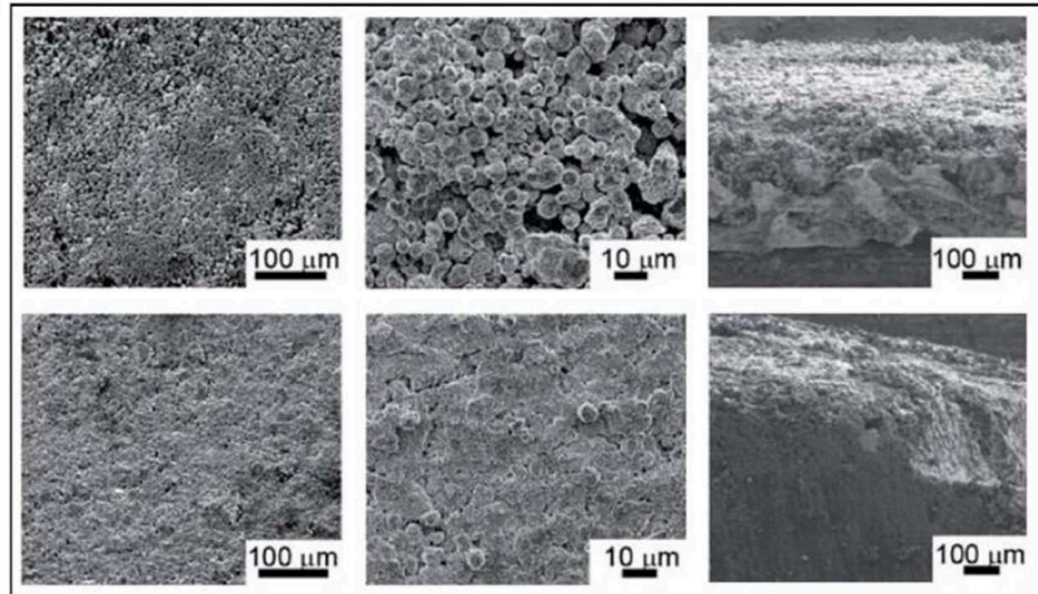

FIGURE 5 | (A) Li stripping and plating process of Li foil and Li-CNT electrodes. (B) Electrochemical impedance spectroscopy of Li||Li (left) and Li-CNT||Li (right) cells at different cycles (below: the equivalent circuit used for curve fitting). (C) Voltage profiles for stripping/plating cycles of Li-CNT\|Li (red line) and Li $\| \mathrm{Li}$ (black line) cells at different current densities (capacity is $20 \mathrm{~mA} \mathrm{~h} \mathrm{~cm}^{-2}$ ). (D) SEM images of the Li foil electrodes and the Li-CNT composite electrodes before and after short-circuit.

Reprinted with permission from Wang Y. et al. (2017). Copyright (2017) Royal Society of Chemistry.

overpotential of the Li-CNT composite electrode is very stable (Figure 5C). The initial dendrite growth causes the surface of the lithium foil to become uneven, increasing the specific surface area of the lithium metal and resulting in a decrease in interface resistance, which corresponds to the results of electrochemical impedance spectroscopy (Figure 5B). From the SEM images, one can observe that the lithium foil is almost entirely destroyed and the Li-CNT composite electrode still preserves its original morphology after cycling (Figure 5D). Therefore, CNTs play a significant role in lithium metal protection.

\section{Graphene}

Graphene is a two-dimensional carbon nanomaterial composed of carbon atoms in an $\mathrm{sp}^{2}$ hybrid orbital hexagonal honeycomb crystal lattice. The chemical properties of graphene are similar to those of graphite, and it can adsorb and desorb various atoms and molecules. When these atoms or molecules act as donors or acceptors, the concentration of graphene carriers can be changed while the graphene itself maintains good conductivity. When other substances are adsorbed, such as $\mathrm{H}^{+}$and $\mathrm{OH}^{-}$, some derivatives are produced that decrease the conductivity of graphene. Because graphene has good electrical conductivity, chemical stability in contact with electrolytes, and mechanical elasticity, it is widely used as a lithium storage medium by researchers (Xin-Bing et al., 2015; Kang et al., 2016; Lin et al., 2016; Zhang R. et al., 2016). Interestingly, Yan et al. (2014) grew two-dimensional (2D) atomic crystal layers, including hexagonal boron nitride (h-BN) and graphene, directly on Cu metal current collectors as an artificial SEI layer (Figure 6A). Moreover, using 3D monolithic corrugated graphene on nickel foam (CGNF) for a Li metal electrode suppresses dendrite growth in carbonatebased electrolytes (Kang et al., 2019). Recently, Chen and his team 


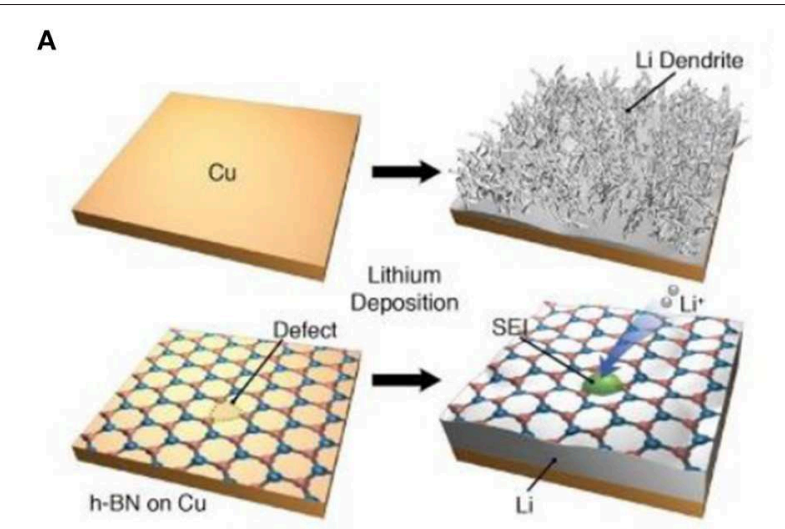

B
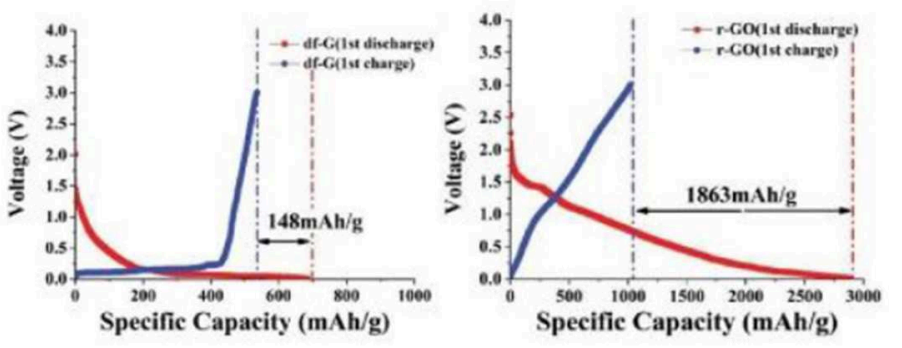

C

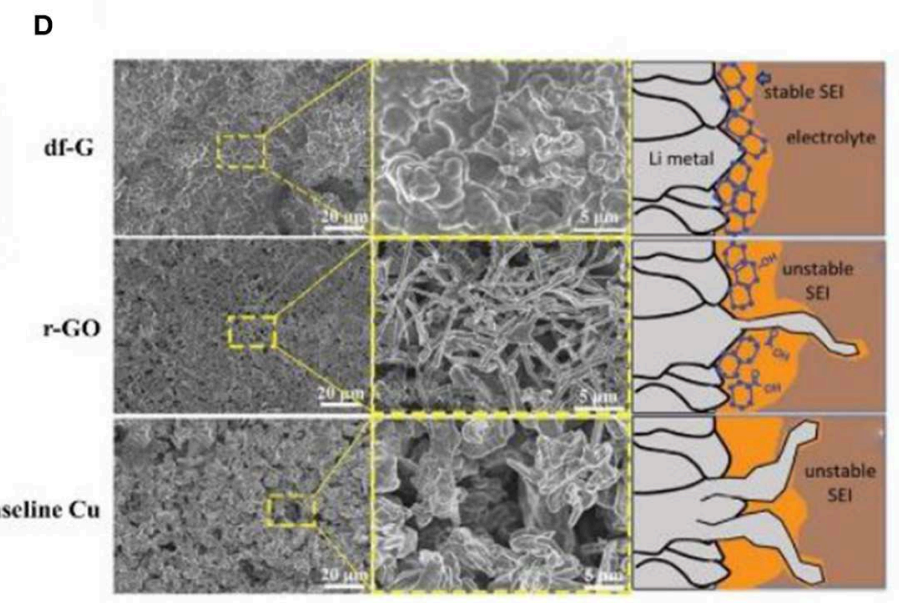

FIGURE 6 | (A) Schematic diagrams of lithium deposition. Reproduced from Yan et al. (2014). Copyright (2014) American Chemical Society (B) Cycle 1 charge/discharge behavior of df-G and $\mathrm{r}-\mathrm{GO}$, respectively. Electrodes were tested in the high voltage range $3 \sim 0.01 \mathrm{~V} \mathrm{vs}$. $\mathrm{Li}^{\prime} / \mathrm{Li}^{+}$, at $100 \mathrm{~mA} \mathrm{~g}^{-1}$. The lower voltage cutoff is significantly above the equilibrium Li plating potential of $\mathrm{OV}$. (C) A comparison of the voltage profiles for df-G, $r-G O$, and bare Cu for the first several plating/stripping cycles at $0.5 \mathrm{~mA} \mathrm{~cm}^{-2}$, with the inset showing the cycle 1 (upper left corner) and cycle 10 (upper right corner) Li metal nucleation potential. Plating/stripping CE at $2 \mathrm{~mA} \mathrm{~cm}^{-2}$ and at various current densities, respectively. (D) Top-down SEM micrographs of the post-100-cycle electrode surfaces in the fully plated condition. Reprinted with permission from Liu et al. (2019). Copyright (2019) WILEY-VCH.

have found that defective graphene contributes to the growth of unstable SEI, resulting in an extremely unstable plating/stripping process (Liu et al., 2019). They adopted a new flow fieldassisted ultrasonic stripping method to strip graphite into defectfree graphene (df-G) and obtained excellent electrochemical performance. They also adopted a test method for the negative electrode of LIBs that could effectively impede the undesirable interaction between the lithium metal and the electrolyte and restrict the initial formation of SEI in $\mathrm{r}-\mathrm{GO}$ and df-G. The irreversible capacity loss in the first cycle for $\mathrm{r}-\mathrm{GO}$ was $1,863 \mathrm{~mA}$ $\mathrm{g}^{-1}$, while that of df-G was only $148 \mathrm{~mA} \mathrm{~h} \mathrm{~g}^{-1}$ (Figure 6B). The SEI formation on the carbon surfaces with this initial capacity loss. In Figure 6C, the overpotential of the first lap of r-GO is lower than that of df-G, while as the cycling continues this trend is inverted. Therefore, df-G can suppress the formation of dendrites and maintain a high CE, low overpotential, and low nucleation barrier of lithium dendrites after plating/stripping 100 times at a current density of $2 \mathrm{~mA} \mathrm{~cm}^{-2}$. Furthermore, according to Figure 6D, df-G can restrain the Li dendrite growth and form a stable SEI on the surface of Li metal. It is wellknown that nitrogen, oxygen, and silicon are pro-lithic, and many studies have looked into the relationship between the "lithiophilicity" of the template and the chemical nature of the carbon supports (Mukherjee et al., 2014; Zhang et al., 2017). However, some studies have found that pro-lithic groups are not necessarily beneficial. It has been reported that it is easier to grow a thicker SEI around nitrogen-doped carbon (Xu et al., 2017). In a nutshell, compared with carbon nanotubes, graphene is relatively difficult to migrate due to its lamellar structure. Therefore, researchers have improved the mobility of lithium ions in graphene by different methods to constrain the growth of dendrites.

\section{Graphite}

Graphite belongs to the hexagonal system and possesses complete layered cleavage. The cleavage plane is mainly intermolecular. Graphite can be principally classified into several types as follows: (1) natural graphite, (2) artificial graphite (i.e., carbon fiber, 
A
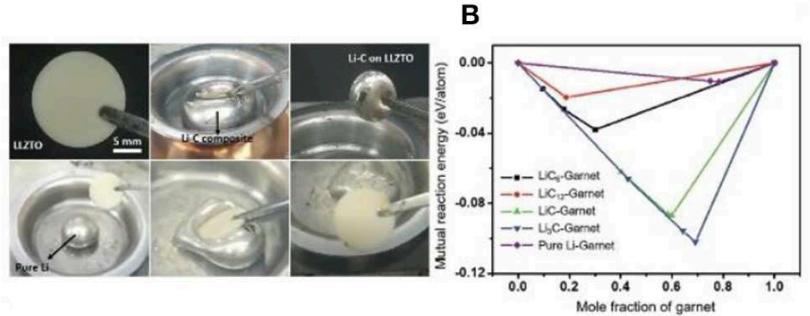

D

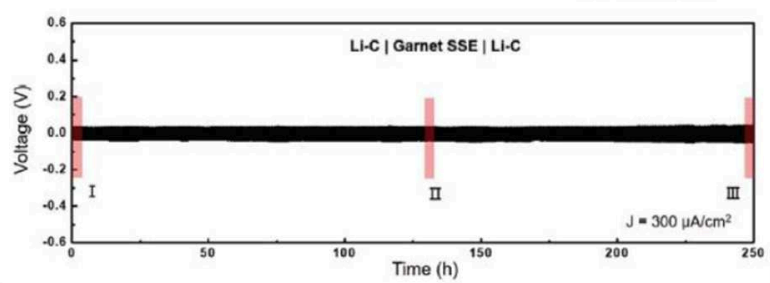

G

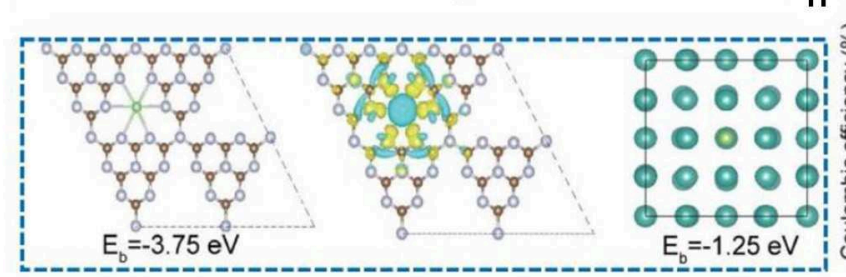

I

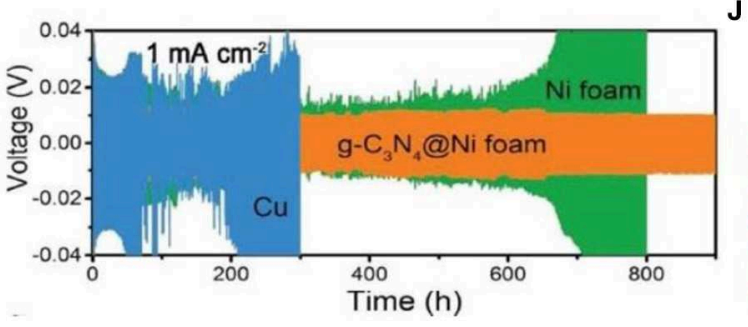

C

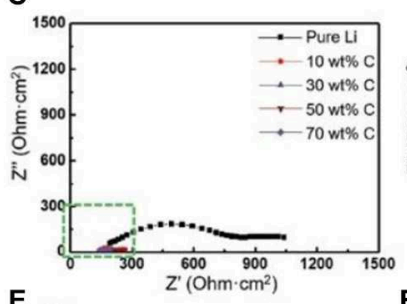

E
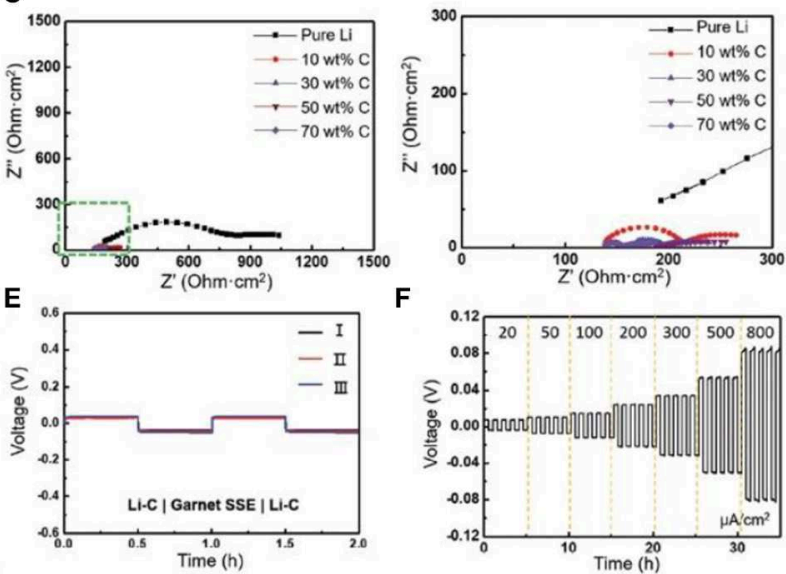

$\mathbf{F}$

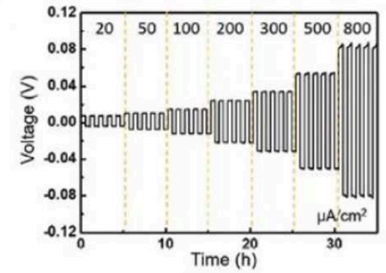

H

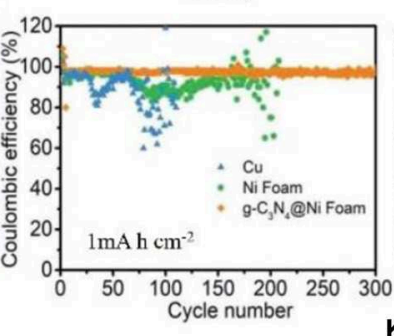

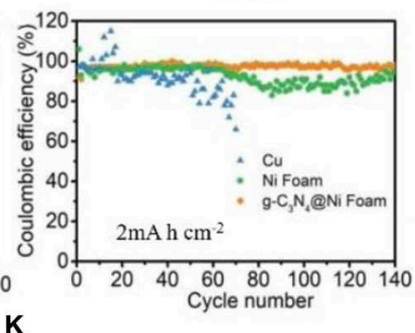
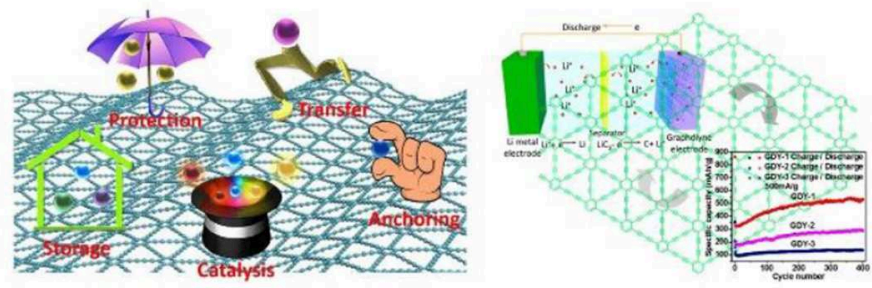

FIGURE 7 | (A) Comparison between pure Li and Li-C lithophilic behaviors with a garnet-type LLZTO pellet. (B) Calculated mutual reaction energy of the Li-C composite/garnet interface and pure Li/garnet interface. (C) Nyquist plots of solid-state symmetric cells with pure Li electrodes and Li-C composite electrodes, where cells with Li-composite electrodes (carbon content from 10 to 70 wt\%) show much smaller interfacial resistance. Reprinted with permission from Duan et al. (2019). Copyright (2019) WILEY-VCH. (D) Reversible plating/striping curves of symmetric-cell Li-C composite electrodes at $300 \mu \mathrm{A} \mathrm{cm}^{-2}$ and room temperature. (E) Three typical charge/discharge curves over long-term cycling as marked in (D). (F) Rate performance of Li-C composite symmetric cells with step-ascending current density from 20 to $800 \mu \mathrm{A} \mathrm{cm}^{-2}$ at room temperature. Reprinted with permission from Duan et al. (2019). Copyright (2019) WILEY- VCH. (G) Crystal models for calculating the binding energy of $\mathrm{Li}^{+}$adsorbed on $\mathrm{g}_{-} \mathrm{C}_{3} \mathrm{~N}_{4}$; the corresponding charge density difference (brown, purple, and green balls represent carbon atoms, nitrogen atoms, and Li atoms, respectively; yellow and light blue areas represent positive and negative charge differences, respectively); crystal models for calculating the binding energy of $\mathrm{Li}^{+}$adsorbed on $\mathrm{Ni}$ (blue and green balls represent nickel atoms and Li atoms, respectively). (H) Comparison of the CE of Li plating on/stripping from $\mathrm{g}-\mathrm{C}_{3} \mathrm{~N}_{4} @ \mathrm{Ni}$ foam, Ni foam, and Cu electrodes with an areal capacity of 1.0 and $2.0 \mathrm{~mA} \mathrm{~h} \mathrm{~cm}^{-2}$ at the same current density of $2.0 \mathrm{~mA}$ cm ${ }^{-2}$. (I) Voltage-time profiles of

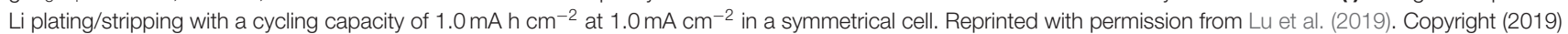
WILEY-VCH. (J) Functions of GDY for electrochemical applications. Reprinted with permission from He et al. (2017). Copyright (2017) Springer Nature Limited. (K) Schematic illustration of a lithium-ion battery with GDY as the electrode and its cycling stability performance. Reprinted with permission from Huang et al. (2015). Copyright (2015) Elsevier Science BV.

pyrolytic carbon, foamed graphite, etc.), (3) bulk graphite, (4) flake graphite, and (5) cryptocrystalline graphite. Due to the good electrical conductivity, plasticity, and chemical stability of graphite, it is widely used in the field of Li metal anode protection. In a recent study, a lithium-graphite ( $\mathrm{Li}-\mathrm{C})$ composite anode was fabricated that reduces the fluidity and increases the viscosity of Li metal and greatly improves the wettability of garnet solidstate electrolytes (Figure 7A) (Duan et al., 2019). According to first-principles calculation, $\mathrm{Li}-\mathrm{C}$ composites can improve the interface affinity between $\mathrm{Li}$ and solid electrolytes (Figure 7B), and when the graphite content increases, the charge transfer resistance gradually decreases, delivering good cycle stability and rate performance (Figure $\mathbf{7 C}$ ). We can see that the voltage hysteresis increases rapidly and the battery fails in a few hours. In comparison, a symmetric cell with a $\mathrm{Li}-\mathrm{C}$ composite electrode shows long-term stability with a small voltage hysteresis over hundreds of hours (Figure 7D). Zoomed-in curves in Figure 7E provide further evidence of overlapped ultraflat plating/striping profiles during long-term cycling, indicating a stable interface between Li-C and garnet SSE. Apart from its stability, the 
Li-C/garnet SSE/Li-C cell also exhibits great rate capability. As shown in Figure 7F, the plating/striping curves are stable as the current density increases from 0.02 to $0.8 \mathrm{~mA} \mathrm{~cm} \mathrm{~cm}^{-2}$, indicating good cycle stability and rate performance. $\mathrm{Lu}$ et al. (2019) reported that graphitic carbon nitride $\left(\mathrm{g}-\mathrm{C}_{3} \mathrm{~N}_{4}\right)$ coated onto foam nickel can form a three-dimensional current collector that can suppress the growth of $\mathrm{Li}$ dendrites and protect the $\mathrm{Li}$ metal anode. It is created by employing super nanosilver (Ag) particles as seeds in the deposition of $\mathrm{Li}$ in a three-dimensional host material, which results in uniform growth of Li (Kai Y. et al., 2016; Pei et al., 2017; Liu et al., 2018). Many studies have shown that local electric field changes can make Li deposition more uniform (Yang et al., 2015; Zou et al., 2018). The 3D current collector designed with a micro-electric field (MEF) by the author can make an even local electric field, leading to even $\mathrm{Li}^{+}$deposition. DFT calculation can reveal the interaction and
A

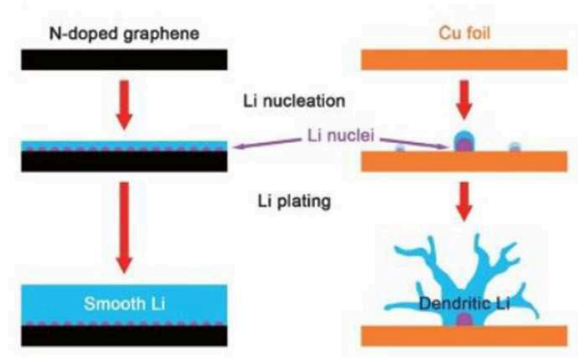

D

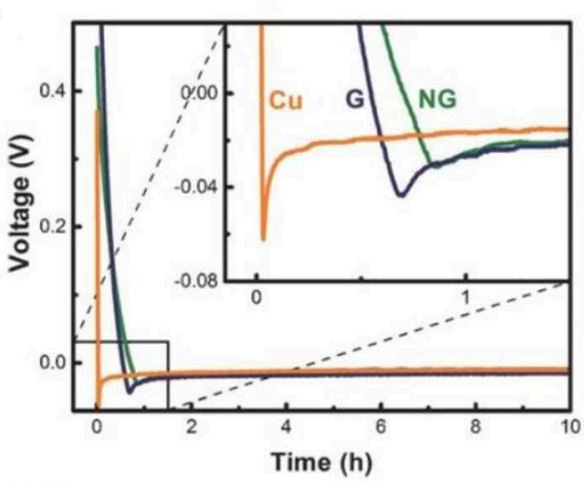

F

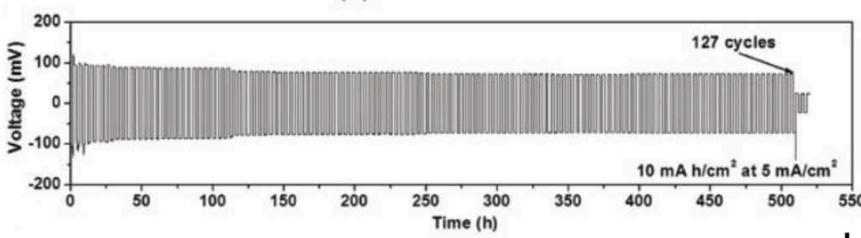

E
B

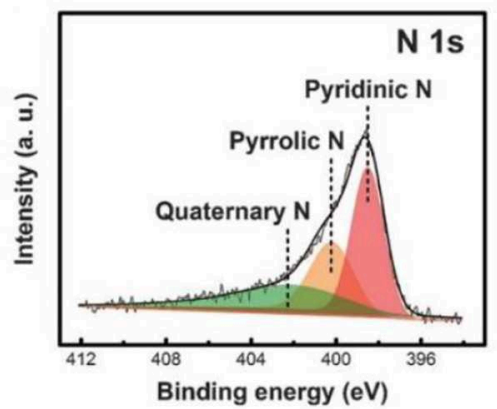

c

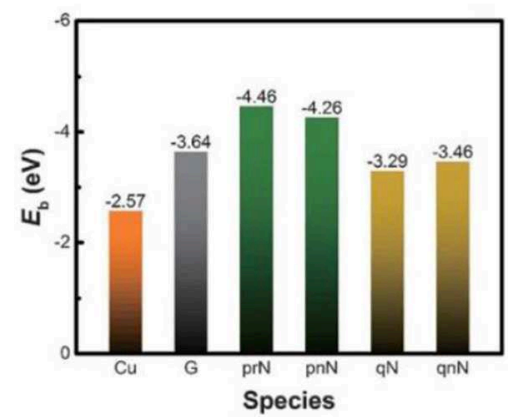

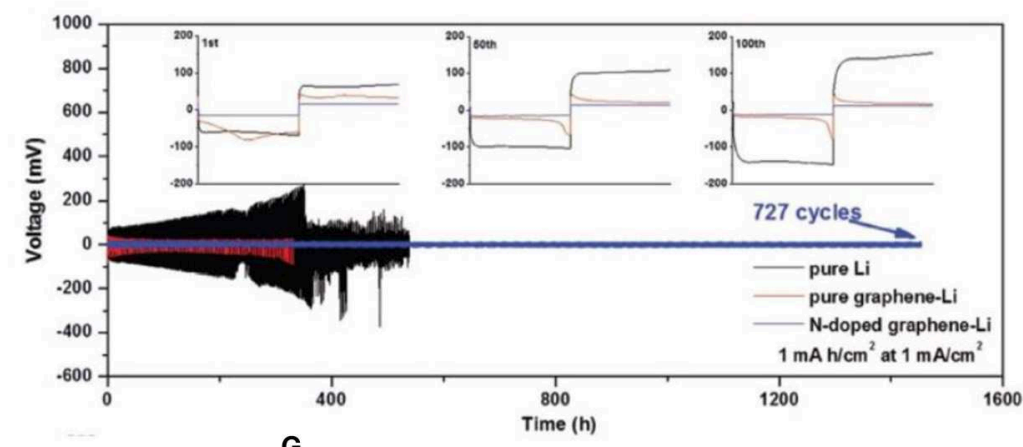

G
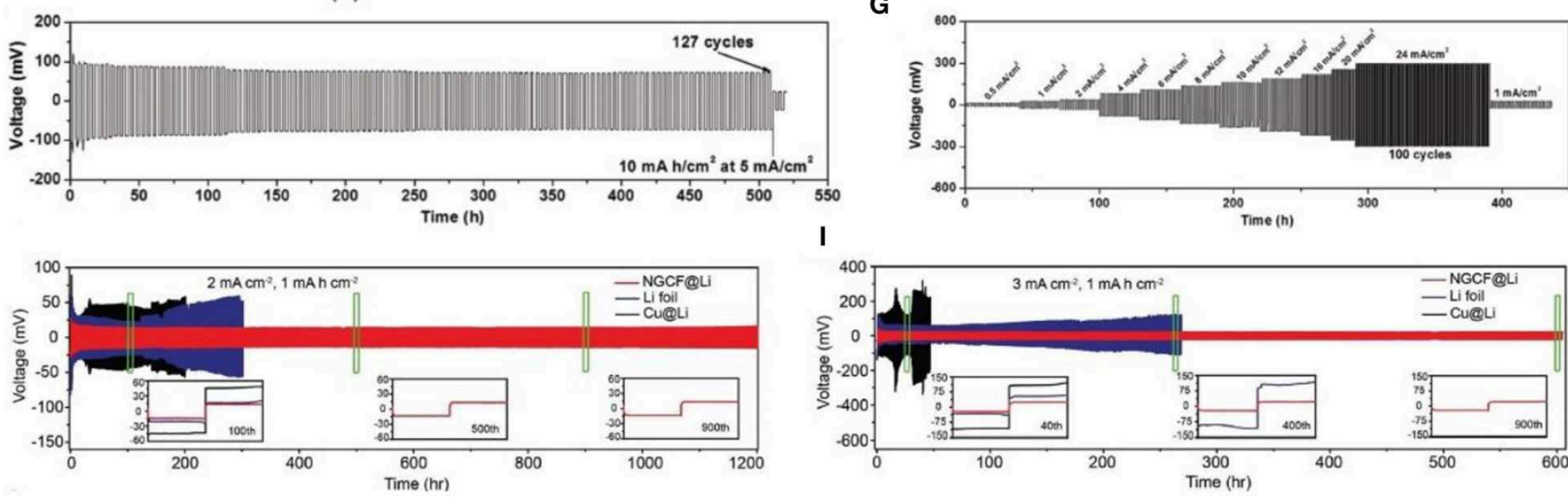

I

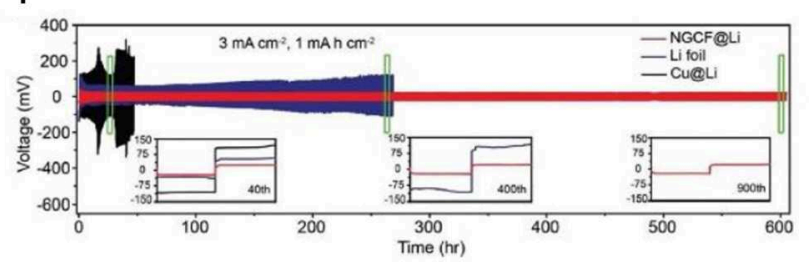

FIGURE 8 | (A) Schematic representation of the Li nucleation and plating process on an N-doped graphene electrode and a Cu foil electrode. (B) N 1s XPS spectra of $\mathrm{N}$-doped graphene. (C) Binding energy of a Li atom with Cu, graphene (G), and different functional groups of N-doped graphene. (D) Nucleation overpotential and the voltage-time curves during Li nucleation at $0.05 \mathrm{~mA} \mathrm{~cm}^{-2}$ on Cu foil, G, and NG electrodes. Reprinted with permission from Zhang et al. (2017). Copyright (2017) WILEY-VCH. Galvanostatic cycling of pure Li foil, pure graphene-Li, and N-doped graphene-Li symmetric cells with a stripping/plating capacity of $1 \mathrm{~mA}$ h $\mathrm{cm}^{-2}$ at a current density of $1 \mathrm{~mA} \mathrm{~cm}^{-2}$. Insets in (E) are the representative voltage profiles. (F) Galvanostatic cycling of symmetric $\mathrm{N}$-doped graphene-Li cells with a stripping/plating capacity of $10 \mathrm{~mA} \mathrm{~h} \mathrm{~cm}^{-2}$ at a current density of $5 \mathrm{~mA} \mathrm{~cm}^{-2}$. (G) Rate performance of N-doped graphene-Li electrodes measured at current densities ranging from 0.5 to $12 \mathrm{~mA} \mathrm{~cm}^{-2}$ for $1 \mathrm{~h}$ and from 16 to $24 \mathrm{~mA} \mathrm{~cm}{ }^{-2}$ at an area capacity of $12 \mathrm{~mA} \mathrm{~h} \mathrm{~cm}^{-2}$ in both the stripping/plating processes of each cycle. Reprinted with permission from Huang et al. (2019). Copyright (2019) WILEY-VCH. (H) Voltage profiles of metallic Li plating/stripping in three styles of symmetric cells (NGCF@Li, Li foil, and Cu@Li cells) at 2 mA cm ${ }^{-2}$ for $1 \mathrm{~mA} \mathrm{~h} \mathrm{~cm}^{-2}$. Inset: magnified Li plating/stripping profiles in the 100th, 500th, and 900th cycles, respectively. (I) Voltage profiles of metallic Li plating/stripping in three styles of symmetric cells at $3 \mathrm{~mA} \mathrm{~cm}^{-2}$ for $1 \mathrm{~mA} \mathrm{~h} \mathrm{~cm} \mathrm{~cm}^{-2}$. Inset: Magnified Li plating/stripping profiles in the 40th, 400th, and 900th cycles, respectively. Reprinted with permission from Liu et al. (2018). Copyright (2018) WILEY-VCH. 
nucleation behavior of $\mathrm{Li}^{+}$and $\mathrm{g}-\mathrm{C}_{3} \mathrm{~N}_{4}$. In this regard, $\mathrm{g}-\mathrm{C}_{3} \mathrm{~N}_{4}$ has the highest binding energy $(-3.75 \mathrm{eV})$ with $\mathrm{Li}^{+}$, while $\mathrm{Ni}$ has a much smaller binding energy $(-1.25 \mathrm{eV})$ (Figure 7G). The g- $\mathrm{C}_{3} \mathrm{~N}_{4} @ \mathrm{Ni}$ foam shows excellent CE (Figure 7H). Moreover, as shown in Figure 7I, the g- $\mathrm{C}_{3} \mathrm{~N}_{4} @ \mathrm{Ni}$ foam anode shows a smaller overpotential at a high amount of $\mathrm{Li}$ deposition (i.e., $9.0 \mathrm{~mA} \mathrm{~h} \mathrm{~cm}^{-2}$ ). In conclusion, the broad application of graphite is due to its good electrochemical performance, but its several shortcomings also limit its further application. Hence, many researchers are attempting to improve its performance through different methods, which is profoundly explored in later discussions.

\section{Graphdiyne}

The three hybrid states of carbon (i.e., $\mathrm{sp}, \mathrm{sp}^{2}$, and $\mathrm{sp}^{3}$ ) are combined in various ways to produce different natural allotropes, such as graphite $\left(\mathrm{sp}^{2}\right)$ and diamond $\left(\mathrm{sp}^{3}\right)$ as well as many synthetic novel carbon allotropes such as fullerene $\left(\mathrm{sp}^{2}\right)$, carbon nanotube $\left(\mathrm{sp}^{2}\right)$, and graphene $\left(\mathrm{sp}^{2}\right)$ (Kroto et al., 1985; Lijima, 1991; Novoselov et al., 2004). In recent years, scientists have been working to explore new forms of carbon allotropes. Accordingly, GDY was first synthesized by Li's group in 2010 (Guoxing et al., 2010), making it a new member of the graphite family. GDY has two hybrid states ( $\mathrm{sp}$ and $\mathrm{sp}^{2}$ ) of carbon. Among the known carbon allotropes, GDY possesses the best mechanical strength and stiffness. Indeed, high conductivity and ion mobility, high chemical activity, and physical stability make GDY an ideal material for solving electrochemical interface problems. Meanwhile, GDY also shows a good affinity with metals (Figure 7J), making it possible to prepare flexible solid electrolytes (Zuo and $\mathrm{Li}, 2019$ ). Its unique structure is more conducive to diffusion and transmission of $\mathrm{Li}^{+}$in and out of the plane, ensuring a good rate performance. In terms of energy storage, GDY is extremely easy to grow on $\mathrm{Si}$ and metal oxide anodes (He et al., 2017), forming an all-carbon mechanically conductive interface, which is advantageous for high-speed transmission of electrons and ions (Shang et al., 2018).

More significantly, the high pore ratio and special structure of GDY provide large diffusion channels and storage sites for metal atoms, such as $\mathrm{Li}$ and $\mathrm{Na}$ (Huang et al., 2018). The range of lithiation potential of GDY is $2.7 \sim 2.1 \mathrm{~V}$, and the intercalation density of lithium is $\mathrm{LiC}_{6}$ in graphite, and $\mathrm{LiC}_{3}$ in Zhang et al. (2011, 2013). In recent researches, Huang et al. (2015) and Zhang et al. (2015) demonstrated that employing GDY in LIBs (Figure 7K) yields superior performance compared to graphite. What is more, the defects on GDY and the introduction of $\mathrm{N}$ (Wang $\mathrm{N}$. et al., 2017), and Cl (Zhang S. et al., 2016) atoms can increase energy storage sites, which is beneficial for reducing interfacial reactions and stabilizing the interface. Note that modification of GDY by doping is also beneficial to electrochemical performance improvement, which is a common modification method for graphene. In a word, GDY shows great prospects in Li metal protection owing to its superior performance (Zuo and Li, 2019). As one of the most commercially valuable materials in the future, GDY will play a huge role in many fields besides the field of electrochemistry.

\section{Doped Carbon Materials}

Although carbon materials have achieved remarkable results in $\mathrm{Li}$ metal protection, there is still a lot of room for development. Currently, there are a variety of methods to modify carbon materials. For instance, lithophilic groups (N, Si, B, F, etc) and materials with solubility ( $\mathrm{Ag}, \mathrm{Zn}, \mathrm{Mg}, \mathrm{Al}, \mathrm{Pt}$, etc) can be introduced by doping, which can change the initial formation behavior of $\mathrm{Li}$ deposition and reduce the nucleation overpotential, thereby restraining the growth of dendrites from the beginning. Moreover, lithophilic groups can increase the specific surface area of the carbon material, decrease local current density, and reduce the nucleation size of Li.

Zhang et al. (2017) used N-doped graphene (NG) to achieve uniform nucleation of Li. Figure 8A presents the nucleation and electroplating process of metallic lithium on N-doped graphenebased bodies and copper current collectors. The N-containing functional groups in NG, including pyridinic $\mathrm{N}$, pyrrolic $\mathrm{N}$, and quaternary $\mathrm{N}$ (Figure 8B), are beneficial to metallic Li nucleation, leading to an even distribution of metal on the surface of the anode (Zhang et al., 2017). According to DFT calculations (Figure 8C), there is a weak interaction between lithium and a copper current collector, while the interaction between pyridine and pyrrole $\mathrm{N}$ is very strong, which is consistent with previous assumptions. In addition, electron donor-rich NG possesses an additional electron pair. When the $\mathrm{P}$ orbital is filled, it can be considered as a Lewis base, which interacts with the Lewis acid $\mathrm{Li}^{+}$in the electrolyte to cause uniform deposition on the lithium surface (Hou et al., 2016). Additionally, it can be seen from the voltage-time curve (Figure 8D) that the nucleation overpotential of NG is the lowest $(0.022 \mathrm{~V})$, while that of $\mathrm{Cu}$ is the highest $(0.053 \mathrm{~V})$. Thus, NG can guarantee a dendrite-free lithium metal anode. However, traditional porous carbon hosts, such as porous carbon nanotubes, and porous graphene provide greater advantages than do porous metals (Zheng et al., 2014; Lin et al., 2016; Zhang R. et al., 2016; Jin et al., 2017; Wang T. et al., 2017; Ye et al., 2017), and the limited surface area cannot effectively attenuate the rate performance of the Li metal anode under high current densities (Zhang R. et al., 2016; Jin et al., 2017; Raji et al., 2017). In recent reports, a nanoporous N-doped graphene-reinforced Li anode (Huang et al., 2019) was applied for Li metal protection. From charging and discharging tests on symmetrical batteries assembled of pure $\mathrm{Li}$, pure graphene-Li, and $\mathrm{N}$-doped graphene (Figure 8E), one can observe that the voltage hysteresis of the $\mathrm{N}$-doped graphene electrode is much smaller than those of the other two electrodes and remains stable after 727 cycles without any obvious voltage fluctuations. It is also evident from the enlarged time-voltage relationship diagram that there are significant differences between the three electrodes. N-doped graphene is significantly better than the other two electrodes. Additionally, it is found that when the deposition capacity and current density are increased, the voltage hysteresis of the $\mathrm{N}$-doped graphene electrode is not meaningful, but the dendrite growth is severe after 127 cycles, piercing the separator, leading to battery short-circuit (Figure 8F). The rate performance test demonstrates that when the current density range is wide, the polarization of the electrode is weakened (Figure 8G). Suffice it to say, owing to the large specific surface 
area and high conductivity of $\mathrm{N}$-doped graphene, it shows great prospects in LMBs.

Furthermore, a self-supporting carbon film (Chen et al., 2019) consisting of a nitrogen (N)-doped carbon rod array was developed for lithium metal protection and achieved remarkable results. N-doped graphitic carbon foams (NGCFs) were also synthesized to suppress dendritic Li growth at the nucleating stage (Liu et al., 2018). According to the timevoltage profiles in Figure 8H, the NGCF@Li symmetrical cell at $2 \mathrm{~mA} \mathrm{~cm} \mathrm{~m}^{-2}$ with $1 \mathrm{~mA} \mathrm{~h} \mathrm{~cm} \mathrm{~m}^{-2}$ exhibits an extremely stable voltage and a long cycle life $(1,200 \mathrm{~h})$, which is superior to the Li foil and Cu@Li symmetrical cell. When the current density is increased to $3 \mathrm{~mA} \mathrm{~cm}^{-2}$ (Figure 8I), the same result is obtained (the NGCF@Li symmetrical cell exhibits stable cycling for $600 \mathrm{~h}$ ). At present, $\mathrm{N}$-doped modified carbon materials are a research hotspot in the field of electrochemistry.

On the basis of $\mathrm{N}$ doping, the performance of F-doped carbon materials has also been investigated. Lately, employing graphite fluoride (GF) (Lazar et al., 2015; Wang et al., 2015) to form a hydrophobic solid-electrolyte interphase on a metalliclithium anode has been explored (Shen et al., 2019). This special anode was prepared by adding graphite fluoride into molten Li, forming a sandwich structure (Figure 9A). In order to prove its hydrophobicity, the Li sheet and GF-LiF-Li composite anode were exposed to the air, and surface changes were
A

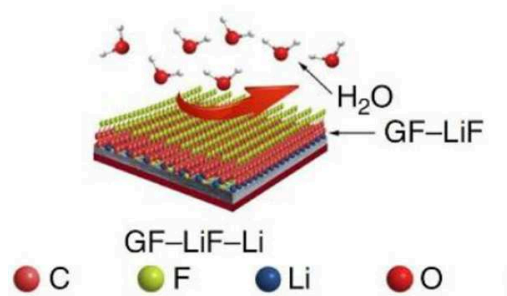

C

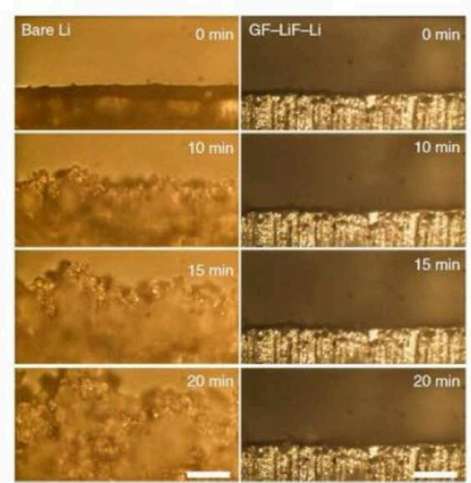

E

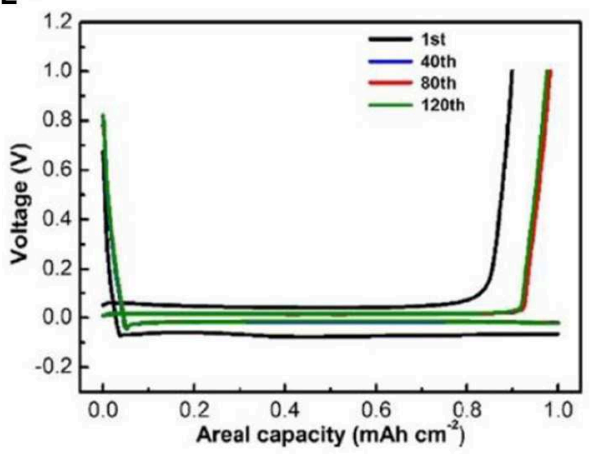

B
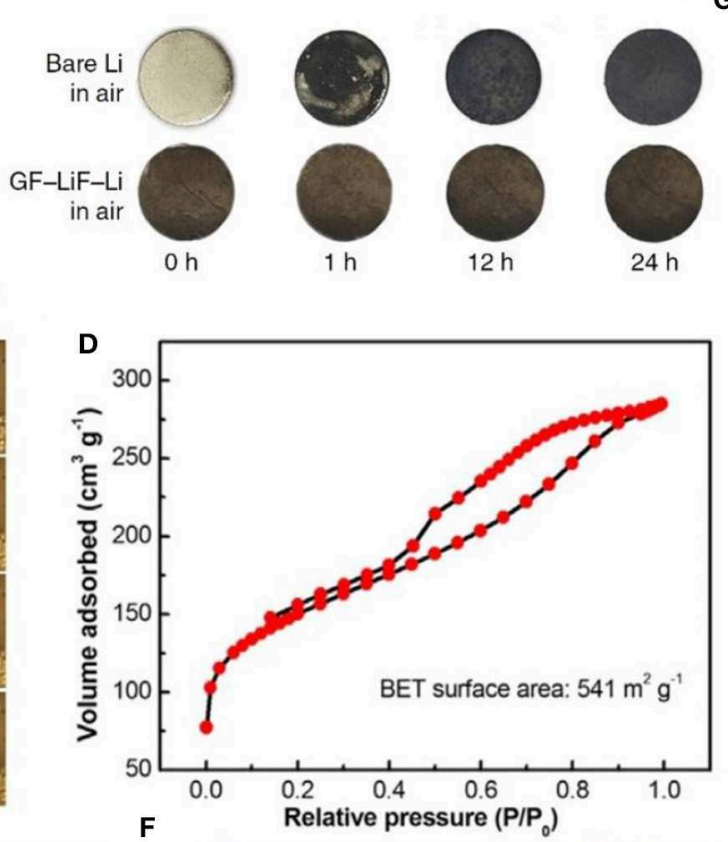

$\mathbf{F}$

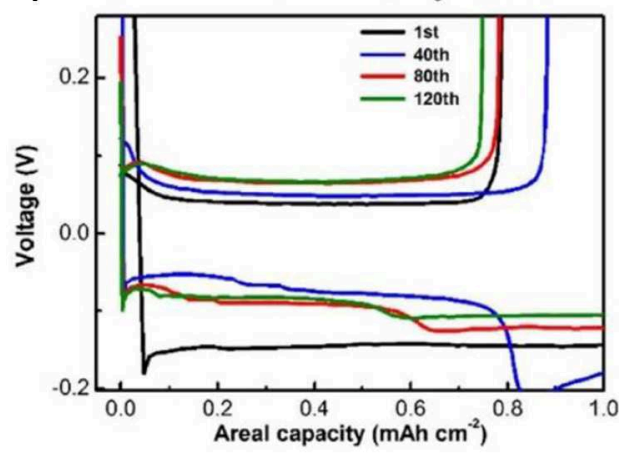

G

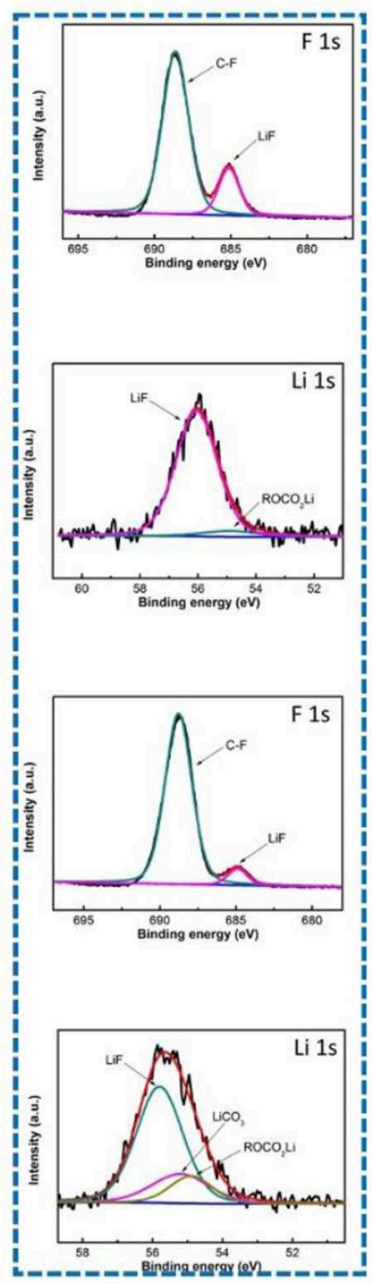

FIGURE 9 | (A) Schematic illustration of the GF-LiF-Li protective effect for Li-metal anodes. In the models, the carbon (C), fluorine (F), lithium (Li), oxygen (O), and hydrogen $(\mathrm{H})$ atoms are displayed as orange, cyan, blue, red, and white spheres, respectively. (B) Characterization of GF-LiF-Li air stability. (C) In-situ optical microscopy visualization of Li electrodeposition and long-term cycling on symmetric cells. Images from microscopy of the bare Li (left column) and GF-LiF-Li (right column) electrolyte interface at $0,10,15$, and $20 \mathrm{~min}$ at a current rate of $3 \mathrm{~mA} \mathrm{~cm}^{-2}$. The scale bars are $200 \mu \mathrm{m}$. Reprinted with permission from Shen et al. (2019). Copyright (2019) Springer Nature Limited. (D) Nitrogen adsorption-desorption isotherms. (E) Voltage profiles of MGCN for the annotated cycles. (F) Voltage profiles of Cu foam for the annotated cycles. (G) XPS graph of LiF-rich SEI, F 1s, and Li 1s XPS spectra of the SEI layer from MGCN and Cu foam electrodes after Li stripping. The results reveal the formation of a LiF-enriched SEl layer on the MGCN electrode upon initial electrochemical lithiation. Reprinted with permission from Li et al. (2018). Copyright (2018) Elsevier Science BV. 
carefully observed. After $24 \mathrm{~h}$, the surface of the Li sheet changed significantly, but the composite electrode did not change significantly (Figure 9B). Therefore, it was proved that a GFLiF-Li composite anode has good stability in the atmosphere. The deposition process of $\mathrm{Li}$ at different times between a pure Li electrode and a GF-LiF-Li electrode was observed by in-situ optical microscopy (Figure 9C), and severe growth of dendrites on the pure Li electrode is clearly noticeable, while no dendrites can be observed on the GF-LiF-Li electrode surface. Thus, the GF-LiF-Li composite anode can suppress dendrite growth to a large extent, providing an opportunity for assembling LMBs in the atmosphere, which substantially reduces production cost. Li et al. (2018) constructed a selfsupporting three-dimensional fluorine-doped graphene shuttleimplanted porous carbon network (MGCN) as a multifunctional host matrix for lithium to inhibit dendrite growth. The addition of graphene can increase the electronic conductivity of MGCN. Its three-dimensional porous structure can improve the mobility of lithium ions. Figure 9D shows that MGCN has a high specific surface area. The voltage distribution profiles of MGCN and of a $\mathrm{Cu}$ foam symmetrical cell were used to verify the structural advantages of MGCN. The electroplating nucleation potential of $\mathrm{Li}$ on $\mathrm{MGCN}$ is $<5 \mathrm{mV}$ (Figure 9E), while that on $\mathrm{Cu}$ foam is more than $40 \mathrm{mv}$ (Figure 9F). Fluorine doping leads to a uniform distribution of lithium ions and the formation of a LiF-rich SEI (proved by XPS test shown in Figure 9G), which is one of the most effective methods for inhibiting dendrite growth.

Oxygen-doped carbon materials have also been widely employed. A Li-rGO electrode was formed by injecting molten Li into a layer of rGO film (Lin et al., 2016), which significantly inhibited the growth of dendrites. It was also found that the molten $\mathrm{Li}$ was in contact with the rGO film and injected into the $\mathrm{rGO}$ at a very fast rate, which was unprecedented among all of the current carbon materials (Figure 10A). The surface of rGO is rich in carbonyl and alkoxy groups (carbonyl is $3.080 \mathrm{eV}$, alkoxy is $2.974 \mathrm{eV}$ ), so the binding energy between $\mathrm{Li}$ and $\mathrm{rGO}$ is much larger (Figure 10B) than that of graphene, which why rGO can absorb the molten Li so quickly. Therefore, rGO has an excellent lithiophilicity, which is related to its scale; the smaller the size, the larger the capillary force (Figure 10C). Figures 10D,E show that during the deposition of lithium, the thickness and volume barely change and no dendrites are visible on the surface. Thus, the rGO film can adjust the volume expansion of Li metal and guide the plating of lithium. Recently, a patterned reduced graphene oxide (P-rGO)/Li anode was also developed (Zou et al., 2018). The electric field around the edge of the patterned electrode can direct lithium ions according to the electric field direction. The voltage profiles of the symmetrical cells were tested at 1,3 , and $10 \mathrm{~mA} \mathrm{~cm}^{-2}$ with $1 \mathrm{~mA} \mathrm{~h} \mathrm{~cm}{ }^{-2}$. The P-rGO/Li anode shows a stable cycle life and relatively low voltage hysteresis compared to metallic lithium and reduced graphene oxide (Figure 10F). The corresponding impedance and rate performance test results are also consistent with the expected results (Figures 10G,H). Additionally, based on the excellent lithiophilic performance and mechanical strength of reduced graphene oxide, Yu's team developed (Yu et al., 2019) a reduced graphene oxide paper to adjust the electroplating process of lithium, and the rapid diffusion of lithium ions was successfully achieved.

\section{Composites With Carbon Materials}

As previously mentioned, carbon-based materials have been widely used in lithium metal protection due to their good electrical conductivity, thermal conductivity, and chemical stability. At present, combining carbon-based materials with other materials to form composites is an effective strategy for lithium metal protection. In this regard, a freestanding porous lithium metal microparticle (Li MP)/carbon nanotube composite anode (LMCA) was prepared (Li et al., 2019a). The electrochemical performance of the half-cell and full-cell assembled with LMCA is significantly better than that with Li foil (Figure 11A). The three-dimensional porous structure of LMCA increases the specific surface area, reduces current density, and alleviates volume expansion. Moreover, a 3D $\mathrm{Cu}-\mathrm{CuO}-\mathrm{Ni}$ conductor ( $\mathrm{Wu}$ et al., 2018), 3D nitrogen-doped graphene foams (Liu et al., 2018), and a 3D porous $\mathrm{Cu}$ collector (Li et al., 2017) have also been employed to protect the $\mathrm{Li}$ metal anode. However, the framework has not fully met the requirements, i.e., it is either too heavy (Chi et al., 2017) or has unsatisfactory lithiophilicity. Recently, a three-dimensional carbon felt (CFelt) coated with a copper oxide layer was synthesized (Yue et al., 2019) that can effectively inhibit dendrite growth and improve the electrochemical performance of the batteries. The $3 \mathrm{D}$ CFelt conductive skeleton has good flexibility and excellent electrical conductivity, allowing it to perfectly alleviate volume expansion, reduce local current density, and improve Li stripping/plating ability (Chen et al., 2017). Cu nanoparticles can further reduce current density and synergize with conductive carbon felt. Therefore, as shown in Figure 11B, a CFeltCu-Li anode has a low voltage hysteresis and long cycle life compared with a pure $\mathrm{Li}$ anode, which attributes to the $\mathrm{CFeltCu}$ with preferable lithiophilicity that can reduce the nucleation overpotential of $\mathrm{Li}$ and impede the formation of dendrites from the beginning. Besides, as depicted in Figure 11C, as time passes, the accumulation of "dead Li" significantly increases the thickness of the bare lithium. On the contrary, there is no "dead Li" accumulation and consequently no change of thickness on the CFeltCu-Li electrode.

Liang et al. (2016) manufactured a stable composite electrode that was prepared by pouring molten lithium into a $3 \mathrm{D}$ porous carbon matrix. The Si-coated porous object can stabilize the electrode/electrolyte interface, reduce the overpotential of Li nucleation, increase the surface area, decrease the local current density, and improve the electrochemical performance. Moreover, a $\mathrm{Li} / \mathrm{C}$ anode has excellent interface stability during cycling (Duan et al., 2013; Kang et al., 2015), and the overpotential is relatively small compared with a pure Li anode (Figure 11D). The Li/C anode can greatly alleviate the volume expansion of $\mathrm{Li}$, which plays a positive role in suppressing dendrite growth. Moreover, a $\mathrm{Co}_{3} \mathrm{O}_{4}$ nanofiber carbon sheet $\left(\mathrm{Co}_{3} \mathrm{O}_{4}\right.$-CS $)$ skeleton with excellent pro-sodium/pro-lithium performance was reported by Li et al. (2019b) that can act as a stable matrix for an alkali metal electrode. They revealed that the 


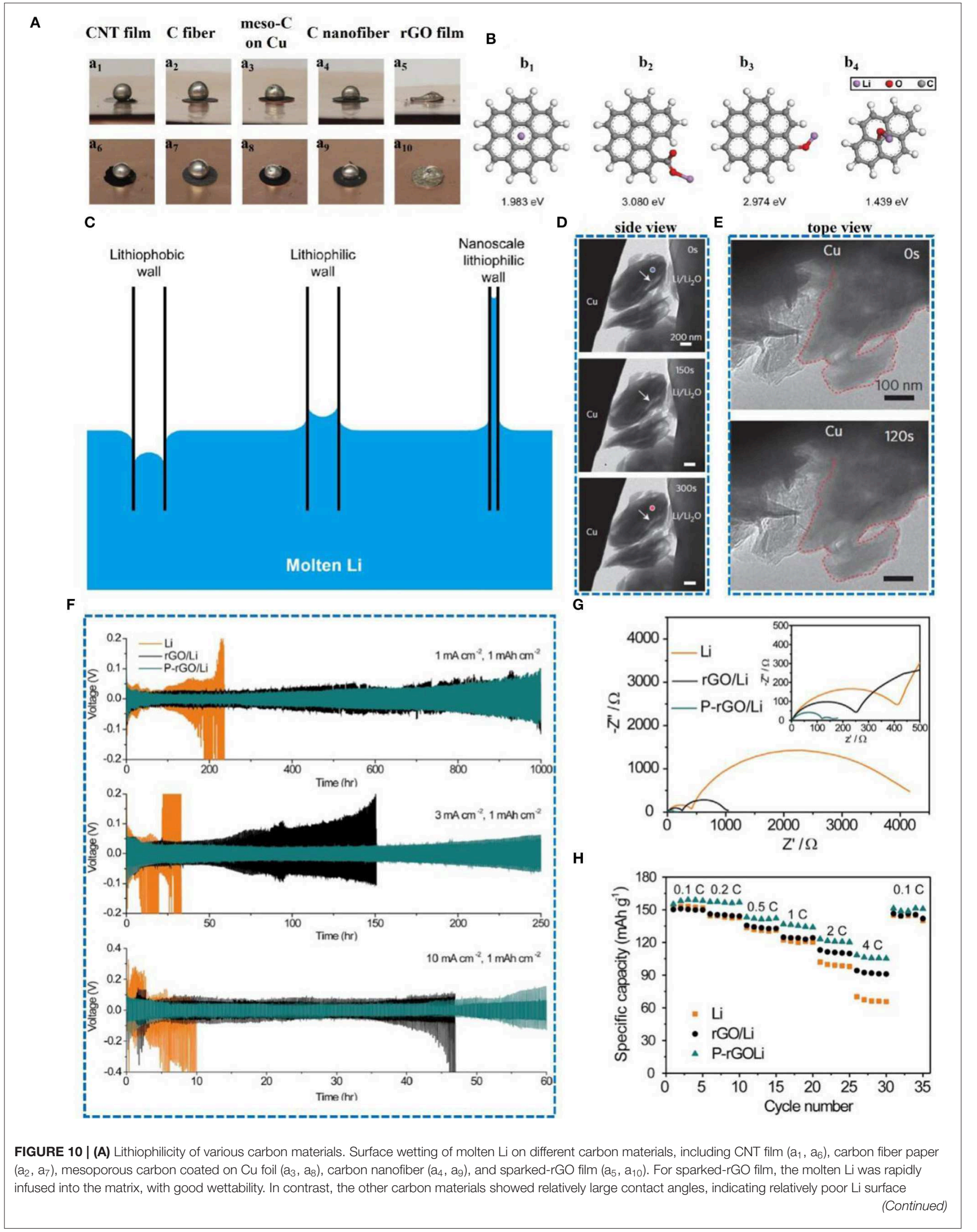


FIGURE 10 | wettability. (B) First-principles calculations showing the binding energy between Li and a bare graphene surface $\left(\mathrm{b}_{1}\right)$, carbonyl $(\mathrm{C}=\mathrm{O})$ groups $\left(\mathrm{b}_{2}\right)$, alkoxy groups (C-O) $\left(b_{3}\right)$, and epoxyl (C-O-C) groups $\left(b_{4}\right)$. The carbonyl and alkoxy groups show much stronger interaction with Li compared to a bare graphene surface. (C) Capillary force at different scales and lithiophilicities. (D) Time-lapse images showing a side view of an rGO film during the Li deposition process. (E) Top view of an rGO film at the initial (top) and final (bottom) stages of Li deposition. Reprinted with permission from Lin et al. (2016). Copyright (2016) Springer Nature Limited. (F) Symmetric cell-cycling performance of Li foil, $\mathrm{rGO} / \mathrm{Li}$, and P-rGO/Li under 1, 3, and $10 \mathrm{~mA} \mathrm{~cm}{ }^{-2}$. The areal capacity was fixed at $1 \mathrm{~mA} \mathrm{~h} \mathrm{~cm}{ }^{-2}$. (G) Electrochemical impedance spectroscopy of symmetric cells of Li foil, rGO/Li, and P-rGO/Li before electrochemical testing. (H) Rate capability of LFP|Li, LFP|rGO/Li, and

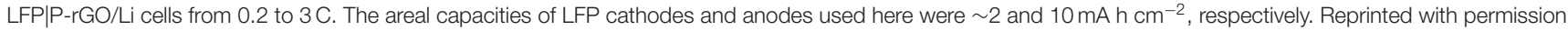
from Wang et al. (2018). Copyright (2018) Elsevier Science BV.

pro-lithium performance of metal oxides has a relationship with the thermodynamics of chemical reactions and thus that $\mathrm{Co}_{3} \mathrm{O}_{4}-$ CS induces good lithiophilicity and uniform nucleation. After stripping and plating, the carbon fiber structure can be clearly observed in the SEM images. The surface of the carbon fiber is completely adhered to the Li (Figure 11E), thereby confirming that $\mathrm{Li}^{+}$is uniformly deposited on the surface of the $\mathrm{Co}_{3} \mathrm{O}_{4}{ }^{-}$ $\mathrm{CS} / \mathrm{Li}$ electrode. This innovative method can effectively improve the cycle stability of a Li metal anode and inhibit the appearance of dendrites.

Although the 3D porous structure has a large specific surface area and effectively stores $\mathrm{Li}$ to alleviate the volume expansion of Li metal, the large specific surface area means that the contact area with the electrolyte is also increased (Liang et al., 2017), and the irreversible reaction on the electrode is amplified. This not only causes a degradation in electrolyte performance but also produces products such as $\mathrm{Li}_{2} \mathrm{CO}_{3}$, lithium alkyl carbonate, and alkyl lithium decomposition, covering the electrode surface and hindering further electron/mass transfer (Lee et al., 2013; Deng et al., 2018a; Jiao et al., 2018). The abovementioned phenomenon has been reported in many studies (Deng et al., 2018b; Wu et al., 2018; Zhang R. et al., 2018). Recently, a surface-engineered coating was developed (Deng W. et al., 2019) with solid polymer electrolyte (SPE) containing polyethylene oxide (PEO) as the polymer matrix, lithium bis (trifluoromethanesulfonyl) imide (LiTFSI) as the Li salt, and graphene oxide (GO) sheets as an enhancing filler (Figure 11F). The ultrathin and conformal SPE coating layer (uSPE) on Ni foam presents a large charge transfer resistance $(0.5 \Omega)$ compared to pure $\mathrm{Ni}$ foam $(0.375 \Omega)$, which can affect the migration of lithium ions. The uniform distribution of $\mathrm{O}$ and $\mathrm{F}$ on the surface of the Ni foam proves the smoothness of the USPE coating (Figure 11G). This is consistent with the original conjecture that evenly distributed the uSPE coating allows $\mathrm{Li}^{+}$to conduct quickly while avoiding electrolyte contact with metallic lithium, reducing the probability of electrolyte decomposition and side reactions.

Notably, researchers have only focused on the large specific surface area of the 3D current collector, which greatly alleviates the volume expansion of metallic lithium, and have ignored its drawbacks. It is worth mentioning that the large specific surface area increases the probability of contact between metallic lithium and the electrolyte and subsequently increases irreversible interfacial reaction of the electrode/electrolyte and has a negative effect on preventing dendrite growth. Accordingly, it is necessary to modify the surface of the $3 \mathrm{D}$ current collectors via other approaches.

\section{THE APPLICATION OF LITHIUM METAL ANODES}

Carbon-based materials are widely used in lithium metal protection owing to their superior thermal and electrical conductivity, high temperature resistance, good mechanical strength, excellent chemical stability, and good lubrication performance. As a result, carbon-based materials can efficiently reinforce materials lithiophilicity, increase lithium ion mobility and ease the volume expansion of lithium metal. The purpose of studying the protection of lithium metal anodes is to pair them with a variety of cathodes and then assemble them into full batteries. Zhao et al. (2018) have investigated carbon paper (CP) as an interlayer to protect lithium metal anodes, which was paired with a $\mathrm{LiFePO}_{4}$ (LFP) cathode to form a full battery. After 380 cycles, compared with the pure Li foil, the capacity can be stabilized at about $130 \mathrm{~mA} \mathrm{~h} \mathrm{~g}^{-1}$. Indeed, in the 2nd cycle and 150th cycle, it shows a small voltage hysteresis (Figure 12A). As shown in Figure 12B, when the Li foil and the Li-CNT electrode are paired with a commercial LFP cathode, the CE can be stabilized at $90.0 \%$ and $90.1 \%$ after 100 and 300 cycles at $0.5 \mathrm{C}$ and $1 \mathrm{C}$, respectively (Wang Y. et al., 2017). Moreover, in a full cell with Li-reduced graphene oxide ( $\mathrm{rGO}$ ) as the anode and $\mathrm{LiCoO}_{2}$ as the cathode (Lin et al., 2016), the battery maintains a high capacity at high rates, especially at $10 \mathrm{C}$, and shows a small overpotential at different rates (Figure 12C) compared with pure Li foil. In another study, a Li@g- $\mathrm{C}_{3} \mathrm{~N}_{4} @ \mathrm{Ni}$ foam and $\mathrm{Li} @ \mathrm{Ni}$ foam composite anodes were paired with a $\mathrm{LiCoO}_{2}$ cathode. The Li@g- $\mathrm{C}_{3} \mathrm{~N}_{4} @ \mathrm{Ni}$ foam $\mid \mathrm{LiCoO}_{2}$ cell exhibited a stable capacity (Figure 12D) and delivered $72.9 \%$ capacity retention (Lu et al., 2019). In a recent article, a GF-LiF-Li anode with good stability in an atmospheric environment was paired with a $\mathrm{LiNiCoMnO}_{2}$ cathode (Shen et al., 2019) and showed a normal charge-discharge curve (Figure 12E). CE was close to $100 \%$, and the capacity fading rate was very low, about $0.06 \%$ per cycle after 300 cycles.

In another study, the author developed a lithiophiliclithiophobic gradient strategy by dripping carbon nanotubes (CNT), with various $\mathrm{ZnO}$ loadings, layer by layer onto $\mathrm{Li}$ foil (GZCNT) (Zhang H. et al., 2018). Li-S batteries were assembled to examine the performance of the GZCNT-coated $\mathrm{Li}$ anode. The cycle stability of GZCNT was significantly improved at $0.2 \mathrm{C}$. After 200 cycles, the remaining capacity of the GZCNT battery was $1.73 \mathrm{~mA} \mathrm{~h} \mathrm{~cm} \mathrm{~cm}^{-2}$ (Figure 13A), which is higher than that of the Li foil $\left(1.12 \mathrm{~mA} \mathrm{~h} \mathrm{~cm}^{-2}\right)$. Hence, a Li foil anode with GZCNT coating is extremely effective for improving the electrochemical performance of Li-S 
A

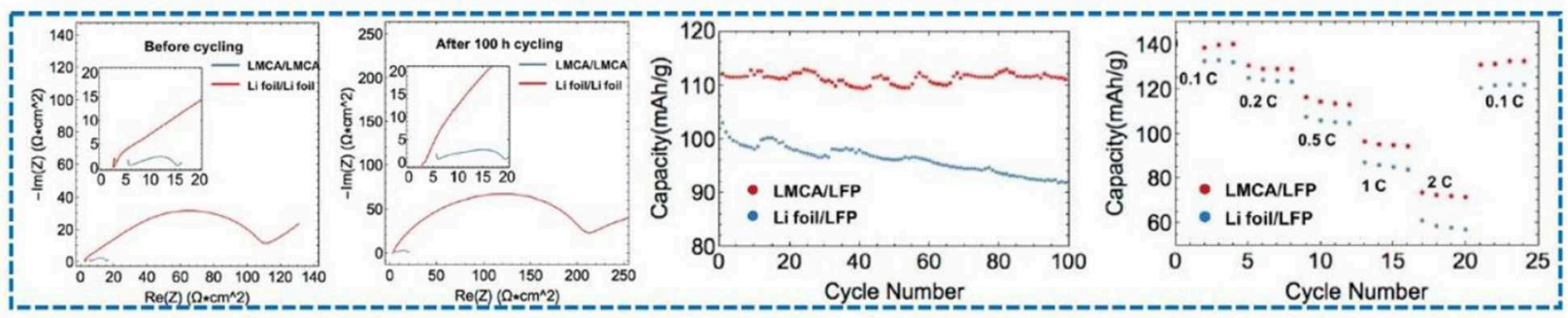

B

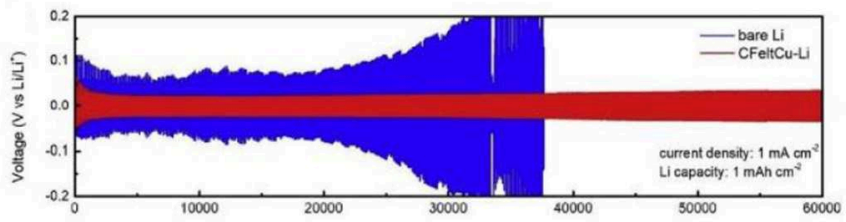

D

C
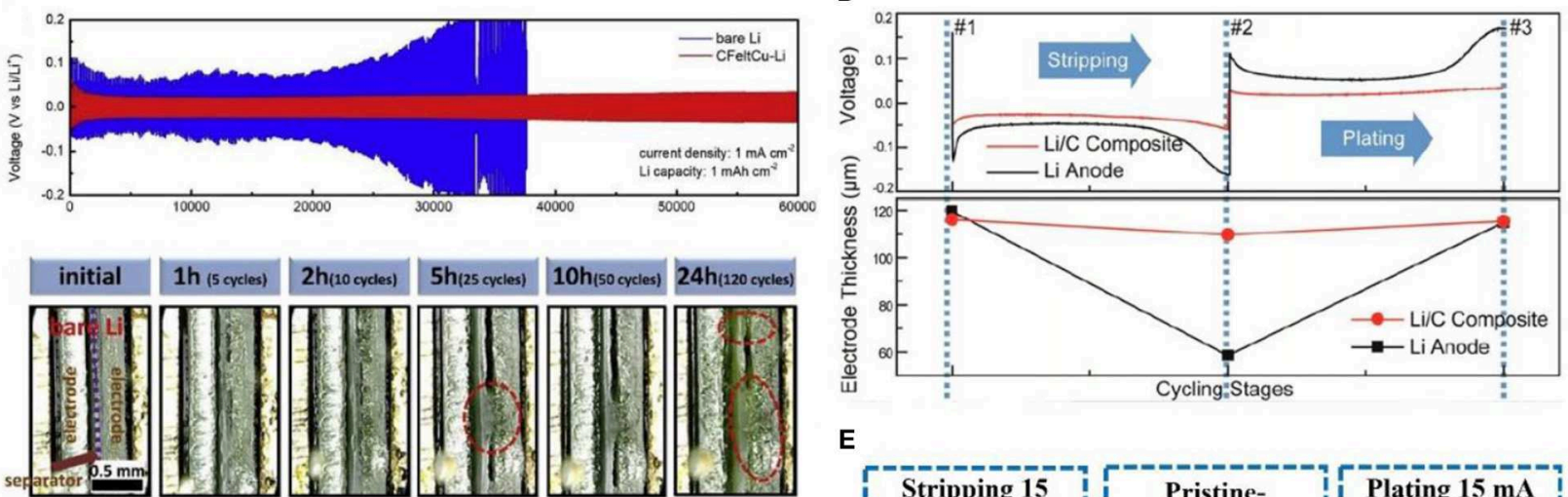

E

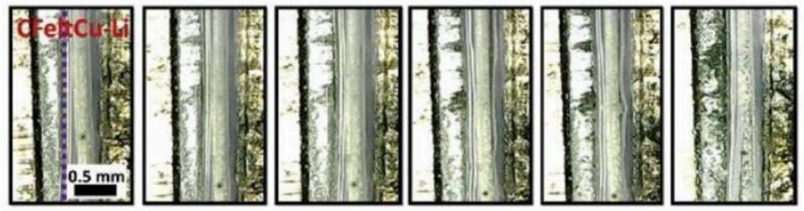

$\mathbf{F}$
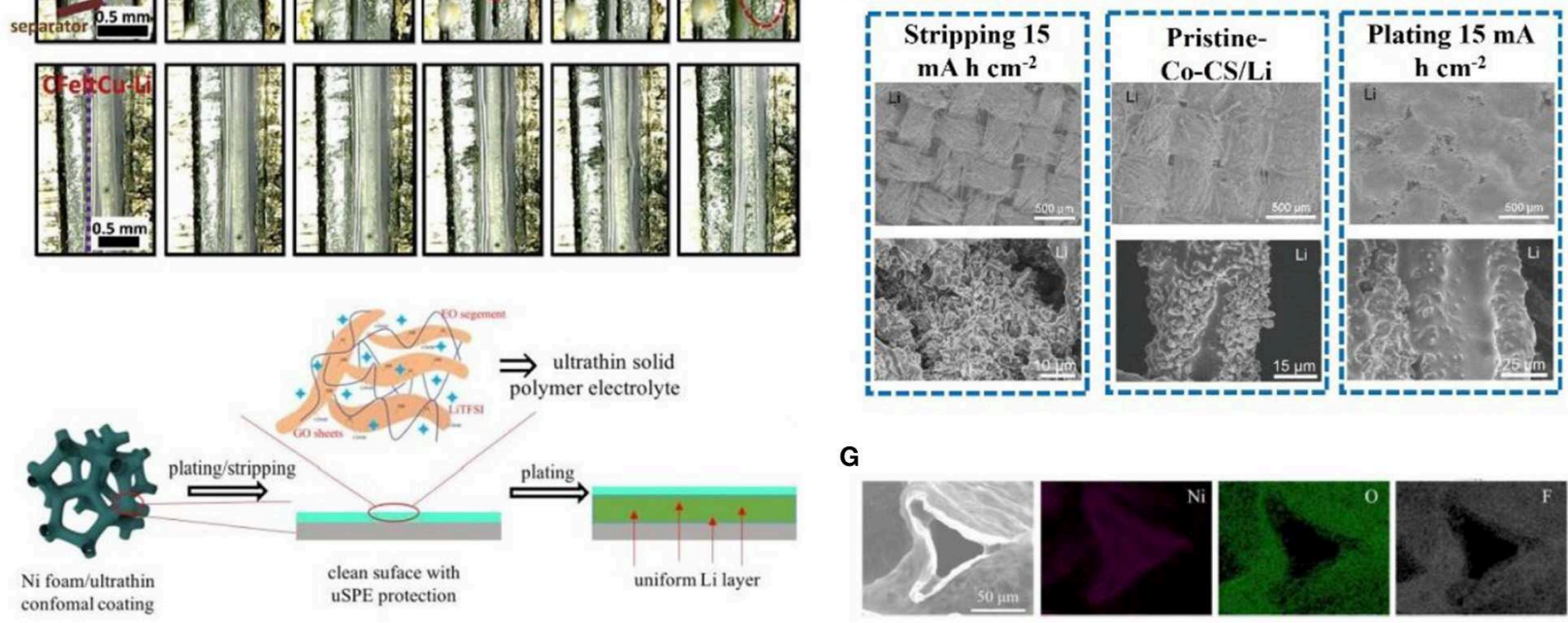

G
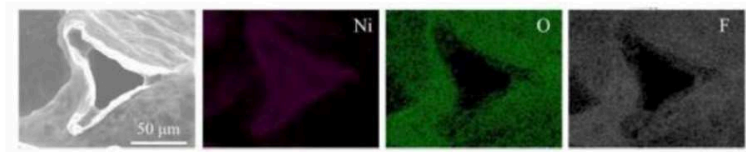

FIGURE 11 | (A) EIS results for LMCA/LMCA and Li foil/Li foil cells at $0.5 \mathrm{~mA} \mathrm{~cm}^{-2}$ before cycling, ElS results for LMCA/LMCA and Li foil/Li foil cells after $100 \mathrm{~h}$ of cycling at $0.5 \mathrm{~mA} \mathrm{~cm}^{-2}$, long-term cycling of LMCA/LFP and Li foil/LFP full cells at $0.5 \mathrm{~mA} \mathrm{~cm}{ }^{-2}$ current density with 2.6 and $3.8 \mathrm{~V}$ voltage cutoffs, and rate capability test of LMCA/LFP and Li foil/LFP full cells at different current densities with 2.6 and $3.8 \mathrm{~V}$ voltage cutoffs. Reprinted with permission from Li et al. (2019a). Copyright (2019a) American Chemical Society. (B) Voltage profile of bare Li and CFeltCu-Li electrodes cycled at $1 \mathrm{~mA} \mathrm{~cm}^{-2}$ with a capacity of $1 \mathrm{~mA} \mathrm{~h} \mathrm{~cm}^{-2}$. (C) Cross-sectional in-situ electron optical microscopy images of the bare Li and CFeltCu-Li electrodes during the cycles at a current density of $10 \mathrm{~mA} \mathrm{~cm}^{-2}\left(\mathrm{capacity}\right.$ is $\left.1 \mathrm{~mA} \mathrm{~h} \mathrm{~cm}^{-2}\right)$. Reprinted with permission from Yue et al. (2019). Copyright (2019) Elsevier Science BV. (D) Voltage profile of a typical Li-stripping-plating process for the anode and its corresponding thickness at various stages of cycling. \#1 corresponds to the stage before cycling; \#2 corresponds to the stage after 50\% Li extraction (10 mA h $\mathrm{cm}^{-2}$ ); \#3 corresponds to the stage after Li plating back. Reprinted with permission from Liang et al. (2016). Copyright (2016) PANS. (E) Low-magnitude SEM images of a Li/Co-CS anode after stripping and plating at $15 \mathrm{~mA} \mathrm{~h} \mathrm{~cm}^{-2} \mathrm{Li}$, and high magnitude SEM images of a single carbon fiber at the different Li deposition periods. Reprinted with permission from Li et al. (2019b). Copyright (2019b) WILEY-VCH. (F) Schematic of the stripping/plating behavior of Li Ni foam with an ultrathin conformal coating. (G) SEM image of Ni foam/USPE and corresponding elemental mapping of Ni, O, and F. Reprinted with permission from Deng W. et al. (2019). Copyright (2019) Royal Society of Chemistry.

batteries. As shown in Figure 13B, Li@g- $\mathrm{C}_{3} \mathrm{~N}_{4} @ \mathrm{Ni}$ foam $\mid \mathrm{S}$ cells were analyzed to verify their potential use in full cells and showed good rate performance and capacity retention (Lu et al., 2019). In a recent study, multi-walled carbon nanotubes (MWCNT) were used as an interlayer in combination with lithium metal as a negative electrode (Deng Y. et al., 2019) and assembled into a $\mathrm{Li}-\mathrm{O}_{2}$ battery to verify the possibility of practical application. Compared with a pure Li electrode, the Li-MWCNT electrode delivers a more stable CE after about 140 cycles (Figure 13C) and has an excellent rate performance. It exhibits a lower voltage hysteresis than bare $\mathrm{Li}$ at all current densities. 
A

B
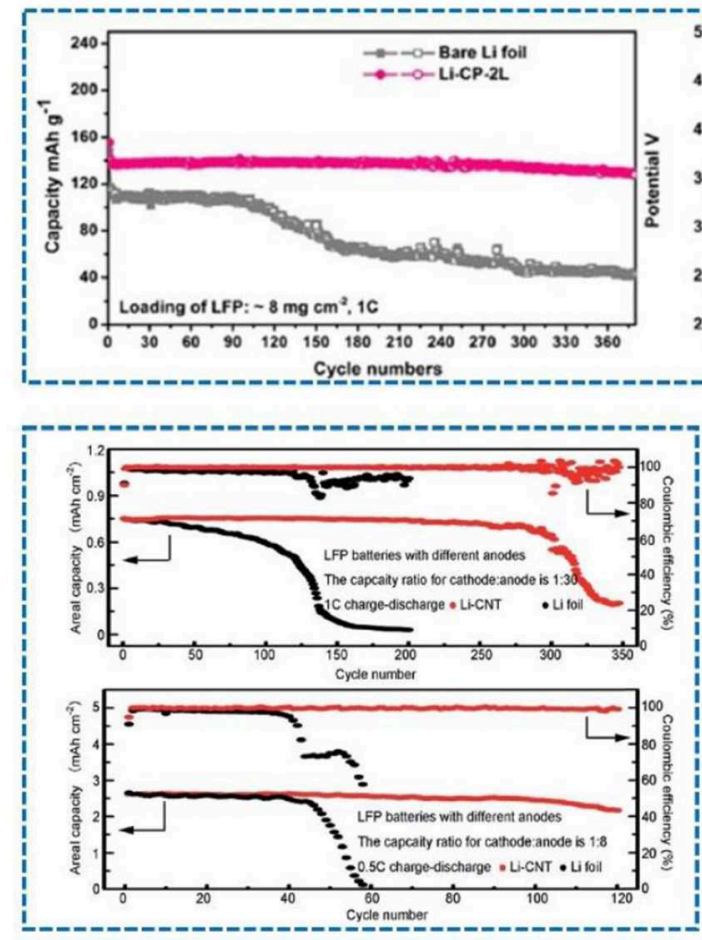

D

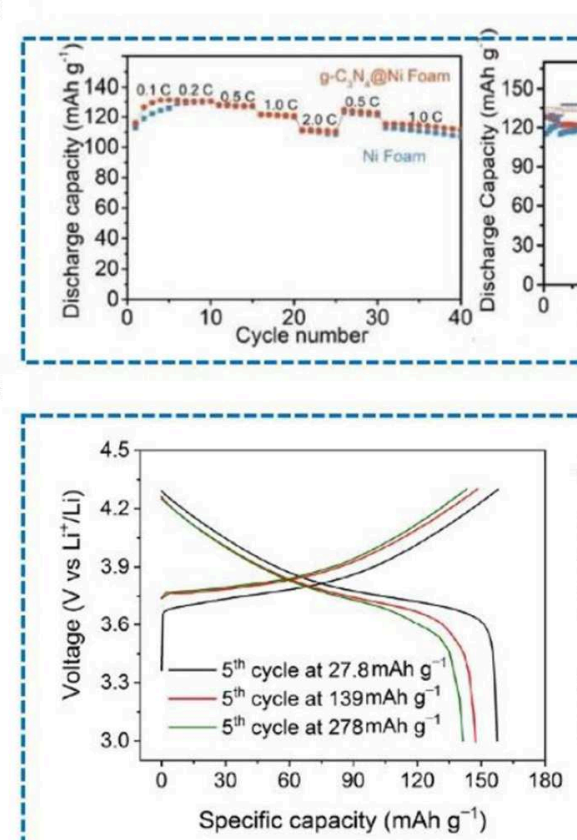

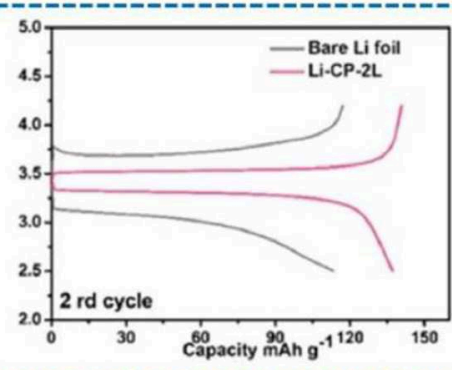

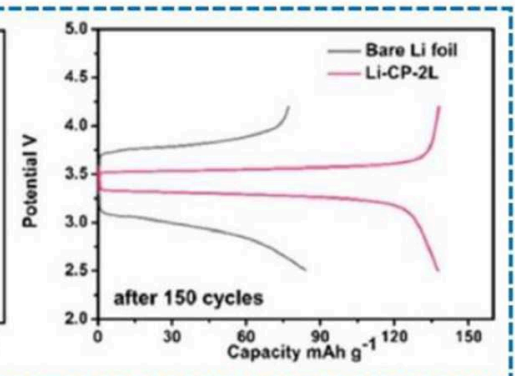

C

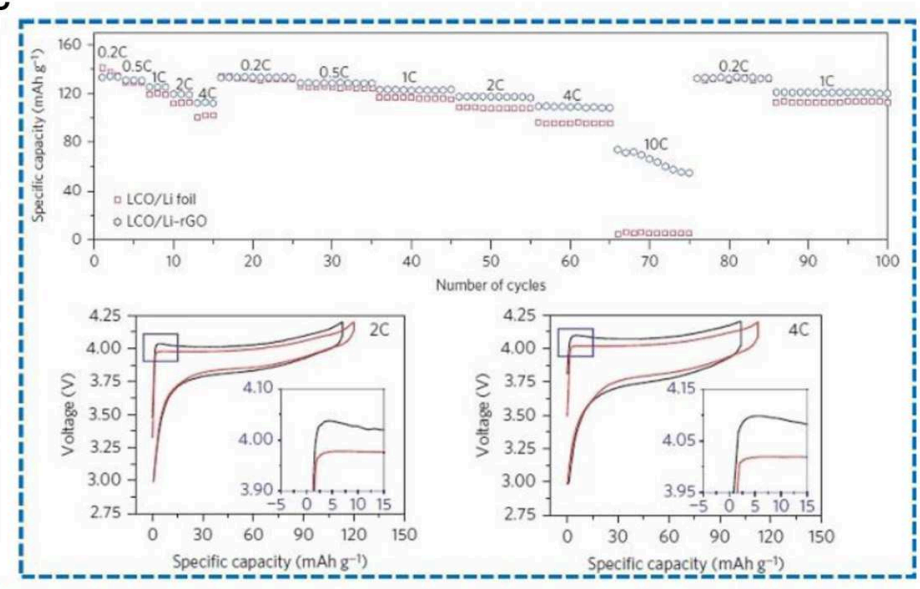

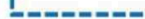

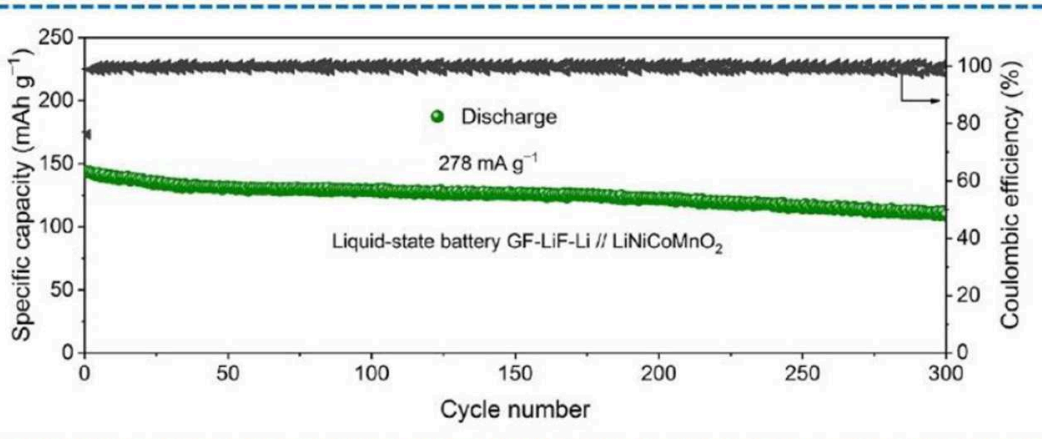

FIGURE 12 | (A) Cycling performance of full cells (C/LiFePO 4 as the cathode) using bare Li foil and Li-CP-2L at 1 C, and voltage hysteresis profiles of charge/discharge of bare Li foil and Li-CP-2L in the 2nd cycle and after the 150th cycle. Reprinted with permission from Zhao et al. (2018). Copyright (2018) Elsevier Science BV. (B) The capacity ratio of cathode to anode is 1:30, and the capacity ratio of cathode to anode is 1:8. Reprinted with permission from Wang Y. et al. (2017). Copyright (2017) Royal Society of Chemistry. (C) Rate capability of LCO/Li-rGO and LCO/Li foil cells at various rates from 0.2 C to $10 \mathrm{C}$, and voltage profile comparison of the LCO/Li-rGO cells at rates of $2 \mathrm{C}$ and 4 C. Reprinted with permission from Lin et al. (2016). Copyright (2016) Springer Nature Limited. (D) Rate

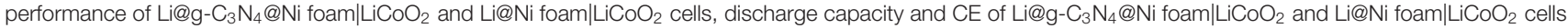
at $1.0 \mathrm{C}$, and charge-discharge profiles of full cells with $\mathrm{LiCoO}_{2}$ as the cathode and $\mathrm{Li} @ \mathrm{~g}-\mathrm{C}_{3} \mathrm{~N}_{4} @ \mathrm{Ni}$ foam $\left(2 \mathrm{~mA} \mathrm{~h} \mathrm{~cm}{ }^{-2} \mathrm{Li}\right.$ deposition) as the anode at $1.0 \mathrm{C}$. Reprinted with permission from Lu et al. (2019). Copyright (2019) WILEY-VCH. (E) The characteristic charge-discharge voltage profiles of liquid-state GF-LiF-Li//LiNiCoMnO 2 cells at different current densities, and a long-term cycling test of GF-LiF-Li//LiNiCoMnO 2 cells at a current density of $278 \mathrm{~mA} \mathrm{~g}^{-1}$. Reprinted with permission from Shen et al. (2019). Copyright (2019) Springer Nature Limited. 


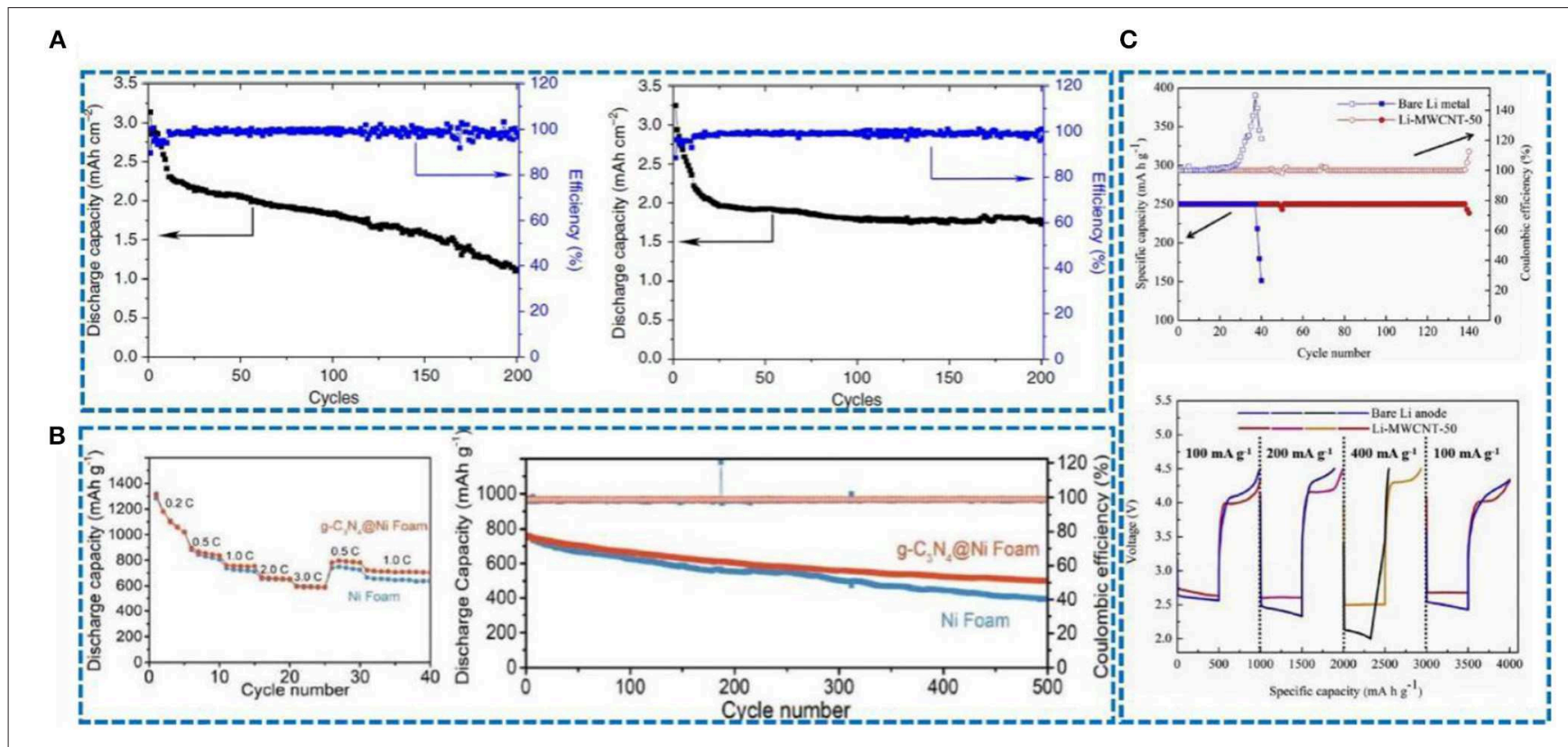

FIGURE 13 | (A) Discharge capacity and coulombic efficiency of Li-S batteries with a pristine Li foil, and discharge capacity and coulombic efficiency of Li-S batteries with a GZCNT-coated Li foil anode. Reprinted with permission from Zhang H. et al. (2018). Copyright (2018) Springer Nature Limited. (B) Rate performance of Li@g-C3N4@Ni foam|S and Li@Ni foam/S cells (1.0C = 1,675 mA g ${ }^{-1}$ for a sulfur cathode), and discharge capacity and CE of Li@g-C3N4@Ni foam|S and Li@Ni foam|S cells at 1.0 C. Reprinted with permission from Lu et al. (2019). Copyright (2019) WILEY-VCH. (C) Cycling stability of the Li- $\mathrm{O}_{2}$ batteries with and without an MWCNT interlayer at a fixed current density of $100 \mathrm{~mA} \mathrm{~g}^{-1}$ and capacity of $250 \mathrm{~mA} \mathrm{~h} \mathrm{~g}^{-1}$ from 2.0 to $4.5 \mathrm{~V}$, and rate capability performances of the $\mathrm{Li}^{\mathrm{O}} \mathrm{O}_{2}$ batteries with and without an MWCNT interlayer at increased current density. Reprinted with permission from Deng Y. et al. (2019). Copyright (2019) Elsevier Science BV.

\section{CONCLUSION AND PERSPECTIVE}

In recent years, low-capacity graphite anodes have no longer been able to meet the needs of human life, and it has become urgent to find appropriate high-capacity anode materials. Hence, a research boom has occurred on the topic of lithium metal anodes. The modification of metallic lithium with a carbon material, in particular, has achieved satisfactory results. Practically, many results have been obtained under laboratory conditions, which have huge prospects for $\mathrm{Li}-\mathrm{S}$ and $\mathrm{Li}-\mathrm{O}_{2}$ batteries in particular. Carbon-based materials possess high conductivity, good chemical stability, and low price and can be used as a good host matrix for accommodating lithium volume expansion and achieving a lighter anode. Although significant effects have been achieved, the inherent instability of metallic lithium in organic electrolytes still needs to be solved urgently. Carbon materials have their own limitations. For instance, their affinity with lithium is not as good as those of metal, metal oxide, and metal sulfide materials, which is a big challenge. In future work, it is inevitable that carbon materials will be utilized in lithium metal protection and that their lithiophilicity will be improved. Due to the complicated internal conditions of the batteries, the mechanism of action for carbon materials

\section{REFERENCES}

Arie, A. A., Vovk, O. M., Song, J. O., Cho, B. W., and Lee, J. K. (2008). Carbon film covering originated from fullerene $\mathrm{C} 60$ on the surface of lithium is still unclear. In addition, $\mathrm{Li}-\mathrm{S}, \mathrm{Li}-\mathrm{O}_{2}$, and all-solid-state lithium batteries are considered to be the most promising nextgeneration energy storage devices. However, there are some issues that need to be resolved first: (1) the poor electrical conductivity of sulfur, and the lithium polysulfide shuttle effect in Li-S batteries, (2) the need for a catalyst for cathode reactions, electrolyte evaporation, electrolyte oxidation, the reactions of moisture and $\mathrm{CO}_{2}$ with lithium metal, and air electrode hole blockage in Li- $\mathrm{O}_{2}$ batteries, (3) the low ionic conductivity of solid electrolytes and poor contact with metallic lithium in all-solid-state lithium batteries. Therefore, multi-faceted research on carbon materials will be very helpful for improving LMB performance.

\section{AUTHOR CONTRIBUTIONS}

All authors wrote the manuscript. XL and SL supervised the manuscript.

\section{ACKNOWLEDGMENTS}

This research was supported by the National Key Research and Development Program of China (No. 2018YFB0105900).

metal anode for lithium secondary batteries. J. Electroceram. 23, 248-253. doi: 10.1007/s10832-008-9413-6

Aurbach, D., Mccloskey, B. D., Nazar, L. F., and Bruce, P. G. (2016). Advances in understanding mechanisms underpinning 
lithium-air batteries. Nat. Energy 1:16128. doi: 10.1038/nenergy.20 16.128

Bai, M.-H., Liu, R., Yang, X.-B., Yu, Z., Wang, Y., and Zhao, Z. (2018). Polypyrrole and manganese oxide composite materials with high working voltage and excellent cycling stability. ChemistrySelect 3, 10574-10579. doi: 10.1002/slct.201802311

Brissot, C., Rosso, M., Chazalviel, J.-N, Baudry, P., and Lascaud, S. (1998). In situ study of dendritic growth inlithium/PEO-salt/lithium cells. Electrochim. Acta 43, 1569-1574. doi: 10.1016/S0013-4686(97)10055-X

Brissot, C., Rosso, M., Chazalviel, J. N., and Lascaud, S. (1999). Dendritic growth mechanisms in lithium/polymer cells. J. Power Sources 81-82, 925-929. doi: 10.1016/S0378-7753(98)00242-0

Bruce, P. G., Freunberger, S. A., Hardwick, L. J., and Jean-Marie, T. (2011). $\mathrm{Li}_{-} \mathrm{O}_{2}$ and Li-S batteries with high energy storage. Nat. Mater. 11, 19-29. doi: 10.1038/nmat3191

Bruce, P. G., and Vincent, C. A. (1987). Steady state current flow in solid binary electrolyte cells. J. Electroanal. Chem. 225, 1-17. doi: 10.1016/0022-0728(87)80001-3

Chazalviel, J. (1990). Electrochemical aspects of the generation of ramified metallic electrodeposits. Phys. Rev. A 42, 7355-7367. doi: 10.1103/PhysRevA.42.7355

Chen, K.-H., Wood, K. N., Kazyak, E., LePage, W. S., Davis, A. L., Sanchez, A. J., et al. (2017). Dead lithium: mass transport effects on voltage, capacity, and failure of lithium metal anodes. J. Mater. Chem. A 5, 11671-11681. doi: 10.1039/C7TA00371D

Chen, L., Chen, H., Wang, Z., Gong, X., Chen, X., Wang, M., et al. (2019). Selfsupporting lithiophilic $\mathrm{N}$-doped carbon rod array for dendrite-free lithium metal anode. Chem. Eng. J. 363, 270-277. doi: 10.1016/j.cej.2019.01.131

Cheng, X. B., and Zhang, Q. (2015). Dendrite-free lithium metal anodes: stable solid electrolyte interphases for high-efficiency batteries. J. Mater. Chem. A 3, 7207-7209. doi: 10.1039/C5TA00689A

Cheng, X. B., Zhang, R., Zhao, C. Z., and Zhang, Q. (2015). Toward safe lithium metal anode in rechargeable batteries: a review. Chem. Rev. 117, 10403-10473. doi: 10.1021 acs.chemrev.7b00115

Chi, S.-S., Liu, Y., Song, W.-L., Fan, L.-Z., and Zhang, Q. (2017). Prestoring lithium into stable 3D nickel foam host as dendrite-free lithium metal anode. $A d v$. Funct. Mater. 27:1700348. doi: 10.1002/adfm.201700348

Deng, W., Liang, S., Zhou, X., Zhao, F., Zhu, W., and Liu, Z. (2019). Depressing the irreversible reactions on a three-dimensional interface towards a highareal capacity lithium metal anode. J. Mater. Chem. A 7, 6267-6274. doi: 10.1039/C9TA00143C

Deng, W., Zhou, X., Fang, Q., and Liu, Z. (2018a). Microscale lithium metal stored inside cellular graphene scaffold toward advanced metallic lithium anodes. $A d v$. Energy Mater. 8:1703152. doi: 10.1002/aenm.201703152

Deng, W., Zhu, W., Zhou, X., and Liu, Z. (2018b). Graphene nested porous carbon current collector for lithium metal anode with ultrahigh areal capacity. Energy Storage Mater. 15, 266-273. doi: 10.1016/j.ensm.2018.05.005

Deng, Y., Lu, H., Cao, Y., Xu, B., Hong, Q., Cai, W., et al. (2019). Multiwalled carbon nanotube interlayers with controllable thicknesses for highcapacity and long-life lithium metal anodes. J. Power Sources 412, 170-179. doi: 10.1016/j.jpowsour.2018.11.037

Ding, F., Xu, W., Graff, G. L., Zhang, J., Sushko, M. L., Chen, X., et al. (2013). Dendrite-free lithium deposition via self-healing electrostatic shield mechanism. J. Am. Chem. Soc. 135, 4450-4456. doi: 10.1021/ja312241y

Duan, B., Wang, W., Zhao, H., Wang, A., Wang, M., Yuan, K., et al. (2013). LiB alloy as anode material for lithium/sulfur battery. ECS Electrochem. Lett. 2, A47-A51. doi: 10.1149/2.005306eel

Duan, J., Wu, W., Nolan, A. M., Wang, T., Wen, J., Hu, C., et al. (2019). Lithiumgraphite paste: an interface compatible anode for solid-state batteries. Adv. Mater. 31:e1807243. doi: 10.1002/adma.201807243

Fleury, V., Chazalviel, J. N., Rosso, M., and Sapoval, B. (1990). The role of the anions in the growth speed of fractal electrodeposits. J. Electroanal. Chem. 290, 249-255. doi: 10.1016/0022-0728(90)87434-L

Giacalone, M., and Nazario, F. (2006). Fullerene polymers:? Synthesis and properties. Chem. Rev. 106, 5136-5190. doi: 10.1002/chin.200718253

Guoxing, L., Yuliang, L., Huibiao, L., Yanbing, G., Yongjun, L., and Daoben, Z. (2010). Architecture of graphdiyne nanoscale films. Chem. Commun. 46, 3256-3258. doi: 10.1039/b922733d
He, J., Wang, N., Cui, Z., Du, H., Fu, L., Huang, C., et al. (2017). Hydrogen substituted graphdiyne as carbon-rich flexible electrode for lithium and sodium ion batteries. Nat. Commun. 8:1172. doi: 10.1038/s41467-017-01202-2

Hong-Jie, P., Jiyuan, L., Lin, Z., Jia-Qi, H., Xin-Bing, C., Xuefeng, G., et al. (2014), Catalytic self-limited assembly at hard templates: a mesoscale approach to graphene nanoshells for lithium-sulfur batteries. ACS Nano 8, 11280-11289. doi: $10.1021 / \mathrm{nn} 503985 \mathrm{~s}$

Hou, T. Z., Chen, X., Peng, H. J., Huang, J. Q., Li, B. Q., Zhang, Q., et al. (2016). Design principles for heteroatom-doped nanocarbon to achieve strong anchoring of polysulfides for lithium-sulfur batteries. Small 12, 3283-3291. doi: $10.1002 /$ smll.201600809

Huang, C., Li, Y., Wang, N., Xue, Y., Zuo, Z., Liu, H., et al. (2018). Progress in research into 2D graphdiyne-based materials. Chem. Rev. 118, 7744-7803. doi: 10.1021 /acs.chemrev.8b00288

Huang, C., Zhang, S., Liu, H., Li, Y., Cui, G., and Li, Y. (2015). Graphdiyne for high capacity and long-life lithium storage. Nano Energy 11, 481-489. doi: 10.1016/j.nanoen.2014.11.036

Huang, G., Han, J., Zhang, F., Wang, Z., Kashani, H., Watanabe, K., et al. (2019). Lithiophilic 3D nanoporous nitrogen-doped graphene for dendritefree and ultrahigh-rate lithium-metal anodes. Adv. Mater. 31:e1805334. doi: 10.1002/adma.201805334

Jiao, S., Ren, X., Cao, R., Engelhard, M. H., Liu, Y., Hu, D., et al. (2018). Stable cycling of high-voltage lithium metal batteries in ether electrolytes. Nat. Energy 3, 739-746. doi: 10.1038/s41560-018-0199-8

Jin, S., Sun, Z., Guo, Y., Qi, Z., Guo, C., Kong, X., et al. (2017). High areal capacity and lithium utilization in anodes made of covalently connected graphite microtubes. Adv. Mater. 29:1700783. doi: 10.1002/adma.201700783

Jiulin, W., Fengjiao, L., Hao, J., Jun, Y., Monroe, C. W., and Yanna, N. L. (2015). Towards a safe lithium-sulfur battery with a flame-inhibiting electrolyte and a sulfur-based composite cathode. Angew. Chem. 126, 10263-10268. doi: 10.1002/ange.201405157

Kai, Y., Lu, Z., Lee, H. W., Feng, X., Hsu, P. C., Li, Y., et al. (2016). Selective deposition and stable encapsulation of lithium through heterogeneous seeded growth. Nat. Energy 1:16010. doi: 10.1038/nenergy.2016.10

Kai, Z., Lee, G. H., Park, M., Li, W., and Kang, Y. M. (2016). Recent developments of the lithium metal anode for rechargeable non-aqueous batteries. Adv. Energy Mater. 6:1600811. doi: 10.1002/aenm.201600811

Kang, H.-K., Woo, S.-G., Kim, J.-H., Lee, S.-R., Lee, D.-G., and Yu, J.S. (2019). Three-dimensional monolithic corrugated graphene/Ni foam for highly stable and efficient Li metal electrode. J. Power Sources 413, 467-475. doi: 10.1016/j.jpowsour.2018.12.075

Kang, H. K., Woo, S. G., Kim, J. H., Lee, S. R., and Kim, Y. J. (2015). Conductive porous carbon film as a lithium metal storage medium. Electrochim. Acta 176, 172-178. doi: 10.1016/j.electacta.2015.06.140

Kang, H. K., Woo, S. G., Kim, J. H., Yu, J. S., Lee, S. R., and Kim, Y. J. (2016). Fewlayer graphene island seeding for dendrite-free li metal electrodes. ACS Appl. Mater. Interfaces 8, 26895-26901. doi: 10.1021/acsami.6b09757

Kroto, H. W., Heath, J. R., O’Brien, S. C., Curl, R. F., and Smalley, R. E. (1985). C60: Buckminsterfullerene. Nature 318, 162-163. doi: 10.1038/318162a0

Lazar, P., Otyepková, E., Karlický, F., Cépe, K., and Otyepka, M. (2015). The surface and structural properties of graphite fluoride. Carbon 94, 804-809. doi: 10.1016/j.carbon.2015.07.064

Lee, J. T., Nitta, N., Benson, J., Magasinski, A., Fuller, T. F., and Yushin, G. (2013). Comparative study of the solid electrolyte interphase on graphite in full Li-ion battery cells using X-ray photoelectron spectroscopy, secondary ion mass spectrometry, and electron microscopy. Carbon 52, 388-397. doi: 10.1016/j.carbon.2012.09.049

Li, Q., Zhu, S., and Lu, Y. (2017). 3D Porous Cu current collector/Li-metal composite anode for stable lithium-metal batteries. Adv. Funct. Mater. 27:1606422. doi: 10.1002/adfm.201606422

Li, S., Liu, Q., Zhou, J., Pan, T., Gao, L., Zhang, W., et al. (2019b). Hierarchical $\mathrm{Co}_{3} \mathrm{O}_{4}$ nanofiber-carbon sheet skeleton with superior $\mathrm{Na} / \mathrm{Li}$-philic property enabling highly stable alkali metal batteries. Adv. Funct. Mater. 29:1808847. doi: $10.1002 / \mathrm{adfm} .201808847$

Li, S., Wang, H., Wu, W., Lorandi, F., Whitacre, J. F., and Matyjaszewski, K. (2019a). Solvent-processed metallic lithium microparticles for lithium metal batteries. ACS Appl. Energy Mater. 2, 1623-1628. doi: 10.1021/acsaem.9b00107 
Li, Z., Li, X., Zhou, L., Xiao, Z., Zhou, S., Zhang, X., et al. (2018). A synergistic strategy for stable lithium metal anodes using 3D fluorinedoped graphene shuttle-implanted porous carbon networks. Nano Energy 49, 179-185. doi: 10.1016/j.nanoen.2018.04.040

Liang, X., Pang, Q., Kochetkov, I. R., Sempere, M. S., Huang, H., Sun, X., et al. (2017). A facile surface chemistry route to a stabilized lithium metal anode. Nat. Energy 2:17119. doi: 10.1038/nenergy.2017.119

Liang, Z., Lin, D., Zhao, J., Lu, Z., Liu, Y., Liu, C., et al. (2016). Composite lithium metal anode by melt infusion of lithium into a 3D conducting scaffold with lithiophilic coating. Proc. Natl. Acad. Sci. U.S.A. 113, 2862-2867. doi: 10.1073/pnas.1518188113

Lijima, S. (1991). Helical microtubules of graphitic carbon. Nature 354, 56-58. doi: 10.1038/354056a0

Lim, H. D., Lee, B., Bae, Y., Park, H., Ko, Y., Kim, H., et al. (2017). Reaction chemistry in rechargeable $\mathrm{Li}_{-} \mathrm{O}_{2}$ batteries. Chem. Soc. Rev. 46, 2873-2888. doi: 10.1039/C6CS00929H

Lin, D., Liu, Y., and Cui, Y. (2017). Reviving the lithium metal anode for highenergy batteries. Nat. Nanotechnol. 12, 194-206. doi: 10.1038/nnano.2017.16

Lin, D., Liu, Y., Liang, Z., Lee, H. W., Sun, J., Wang, H., et al. (2016). Layered reduced graphene oxide with nanoscale interlayer gaps as a stable host for lithium metal anodes. Nat. Nanotechnol. 11, 626-632. doi: 10.1038/nnano.2016.32

Liu, L., Yin, Y. X., Li, J. Y., Wang, S. H., Guo, Y. G., and Wan, L. J. (2018). Uniform lithium nucleation/growth induced by lightweight nitrogen-doped graphitic carbon foams for high-performance lithium metal anodes. Adv. Mater. 30:6216. doi: 10.1002/adma.201706216

Liu, W., Xia, Y., Wang, W., Wang, Y., Jin, J., Chen, Y., et al. (2019). Pristine or highly defective? Understanding the role of graphene structure for stable lithium metal plating. Adv. Energy Mater. 9:1802918. doi: 10.1002/aenm.201802918

Liu, Y., Lin, D., Liang, Z., Zhao, J., Yan, K., and Cui, Y. (2016). Lithium-coated polymeric matrix as a minimum volume-change and dendrite-free lithium metal anode. Nat. Commun. 7:10992. doi: 10.1038/ncomms10992

Lu, Z., Liang, Q., Wang, B., Tao, Y., Zhao, Y., Lv, W., et al. (2019). Graphitic carbon nitride induced micro-electric field for dendrite-free lithium metal anodes. Adv. Energy Mater. 9:1803186. doi: 10.1002/aenm.201 803186

Maleki Kheimeh Sari, H., and Li, X. (2019). Controllable cathode-electrolyte interface of $\mathrm{Li}\left[\mathrm{Ni}_{0.8} \mathrm{Co}_{0.1} \mathrm{Mn}_{0.1}\right] \mathrm{O}_{2}$ for lithium ion batteries: a review. $A d v$. Energy Mater. 2019:1901597. doi: 10.1002/aenm.201901597

Manthiram, A., Fu, Y., Chung, S. H., Zu, C., and Su, Y. S. (2014). Rechargeable lithium-sulfur batteries. Chem. Rev. 114, 11751-11787. doi: 10.1021/cr500062v

Matsuda, S., Kubo, Y., Uosaki, K., and Nakanishi, S. (2017). Lithium-metal deposition/dissolution within internal space of CNT 3D matrix results in prolonged cycle of lithium-metal negative electrode. Carbon 119, 119-123. doi: 10.1016/j.carbon.2017.04.032

Mukherjee, R., Thomas, A. V., Datta, D., Singh, E., Li, J., Eksik, O., et al. (2014). Defect-induced plating of lithium metal within porous graphene networks. Nat. Commun. 5:3710. doi: 10.1038/ncomms4710

Novoselov, K. S., Geim, A. K., Morozov, S. V., Jiang, D., Zhang, Y., and Dubonos, S. V. (2004). Electric field effect in atomically thin carbon films. Science 306, 666-669. doi: 10.1126/science.1102896

Pei, A., Zheng, G., Shi, F., Li, Y., and Cui, Y. (2017). Nanoscale nucleation and growth of electrodeposited lithium metal. Nano Lett. 17, 1132-1139. doi: 10.1021/acs.nanolett.6b04755

Peng, H. J., Hou, T. Z., Qiang, Z., Huang, J. Q., Cheng, X. B., Guo, M. Q., et al. (2015). Batteries: strongly coupled interfaces between a heterogeneous carbon host and a sulfur-containing guest for highly stable lithium-sulfur batteries: mechanistic insight into capacity degradation. Adv. Mater. Interfaces 1:1400227. doi: 10.1002/admi.201400227

Raji, A. O., Villegas Salvatierra, R., Kim, N. D., Fan, X., Li, Y., et al. (2017). Lithium batteries with nearly maximum metal storage. ACS Nano 11, 6362-6369. doi: 10.1021/acsnano.7b02731

Rosso, M., Gobron, T., Brissot, C., Chazalviel, J. N., and Lascaud, S. (2001). Onset of dendritic growth in lithium/polymer cells. J. Power Sources 97, 804-806. doi: $10.1016 / S 0378-7753(01) 00734-0$

Sand, H. J. S. (1901). On the concentration at the electrodes in a solution, with special reference to the liberation of hydrogen by electrolysis of a mixture of copper sulphate and sulphuric acid. Philos. Mag. 1, 45-79. doi: 10.1080/14786440109462590

Shang, H., Zuo, Z., Yu, L., Wang, F., He, F., and Li, Y. (2018). Low-temperature growth of all-carbon graphdiyne on a silicon anode for high-performance lithium-ion batteries. Adv. Mater. 30:e1801459. doi: 10.1002/adma.201801459

Shen, X., Li, Y., Qian, T., Liu, J., Zhou, J., Yan, C., et al. (2019). Lithium anode stable in air for low-cost fabrication of a dendrite-free lithium battery. Nat. Commun. 10:900. doi: 10.1038/s41467-019-08767-0

Suo, L., Hu, Y. S., Li, H., Armand, M., and Chen, L. (2012). A new class of solventin-salt electrolyte for high-energy rechargeable metallic lithium batteries. Nat. Commun. 4:1481. doi: 10.1038/ncomms 2513

Tarascon, J. M., and Armand, M. (2001). Issues and challenges facing. Nat. Energy 414, 359-367. doi: 10.1038/35104644

Wang, A., Zhang, X., Yang, Y.-W., Huang, J., Liu, X., and Luo, J. (2018). Horizontal centripetal plating in the patterned voids of Li/graphene composites for stable lithium-metal anodes. Chem 4, 2192-2200. doi: 10.1016/j.chempr.2018.06.017

Wang, J. N., Zhang, Y. L., Liu, Y., Zheng, W., Lee, L. P., and Sun, H. B. (2015). Recent developments in superhydrophobic graphene and graphene-related materials: from preparation to potential applications. Nanoscale 7, 7101-7114. doi: 10.1039/C5NR00719D

Wang, K., Luo, S., Wu, Y., He, X., Zhao, F., Wang, J., et al. (2013). Super-aligned carbon nanotube films as current collectors for lightweight and flexible lithium ion batteries. Adv. Funct. Mater. 23, 846-853. doi: 10.1002/adfm.201202412

Wang, N., He, J., Tu, Z., Yang, Z., Zhao, F., Li, X., et al. (2017). Synthesis of chlorine-substituted graphdiyne and applications for lithium-ion storage. Angew. Chem. Int. Ed. Engl. 56, 10740-10745. doi: 10.1002/anie.201704779

Wang, T., Villegas Salvatierra, R., Jalilov, A. S., Tian, J., and Tour, J. M. (2017). Ultrafast charging high capacity asphalt-lithium metal batteries. ACS Nano 11, 10761-10767. doi: 10.1021/acsnano.7b05874

Wang, Y., Shen, Y., Du, Z., Zhang, X., Wang, K., Zhang, H., et al. (2017). A lithiumcarbon nanotube composite for stable lithium anodes. J. Mater. Chem. A 5, 23434-23439. doi: 10.1039/C7TA08531A

Whittingham, M. S. (2012). History, evolution, and future status of energy storage. Proc. IEEE 100, 1518-1534. doi: 10.1109/JPROC.2012.2190170

Wu, S., Zhang, Z., Lan, M., Yang, S., Cheng, J., Cai, J., et al. (2018). Lithiophilic $\mathrm{Cu}-\mathrm{CuO}-\mathrm{Ni}$ hybrid structure: advanced current collectors toward stable lithium metal anodes. Adv. Mater. 30:1705830. doi: 10.1002/adma.201705830

Wu, X., Wang, J., Fei, D., Chen, X., Nasybulin, E., Zhang, Y., et al. (2014). Lithium metal anodes for rechargeable batteries. Energy Env. Sci. 7, 513-537. doi: 10.1039/C3EE40795K

Xin-Bing, C., Hong-Jie, P., Jia-Qi, H., Rui, Z., Chen-Zi, Z., and Qiang, Z. (2015). Dual-phase lithium metal anode containing a polysulfide-induced solid electrolyte interphase and nanostructured graphene framework for lithiumsulfur batteries. ACS Nano 9, 6373-6382. doi: 10.1021/acsnano.5b01990

Xu, J., Mahmood, J., Dou, Y., Dou, S., Li, F., Dai, L., et al. (2017). 2D Frameworks of $\mathrm{C} 2 \mathrm{~N}$ and $\mathrm{C} 3 \mathrm{~N}$ as new anode materials for lithium-ion batteries. Adv. Mater. 29:2007. doi: 10.1002/adma.201702007

Yan, K., Lee, H. W., Gao, T., Zheng, G., Yao, H., Wang, H., et al. (2014). Ultrathin two-dimensional atomic crystals as stableinterfacial layer for improvement of lithium metal anode. Nano Lett. 14, 6016-6022. doi: 10.1021/nl503125u

Yang, C. P., Yin, Y. X., Zhang, S. F., Li, N. W., and Guo, Y. G. (2015). Accommodating lithium into 3D current collectors with a submicron skeleton towards long-life lithium metal anodes. Nat. Commun. 6:8058. doi: $10.1038 /$ ncomms 9058

Ye, H., Xin, S., Yin, Y. X., Li, J. Y., Guo, Y. G., and Wan, L. J. (2017). Stable Li plating/stripping electrochemistry realized by a hybrid li reservoir in spherical carbon granules with 3D conducting skeletons. J. Am. Chem. Soc. 139, 5916-5922. doi: 10.1021/jacs.7b01763

Yingying, L., Zhengyuan, T., and Archer, L. A. (2014). Stable lithium electrodeposition in liquid and nanoporous solid electrolytes. Nat. Mater. 13, 961-969. doi: 10.1038/nmat4041

Yu, Y., Huang, W., Song, X., Wang, W., Hou, Z., Zhao, X., et al. (2019). Thermally reduced graphene paper with fast $\mathrm{Li}$ ion diffusion for stable $\mathrm{Li}$ metal anode. Electrochim. Acta 294, 413-422. doi: 10.1016/j.electacta.2018.10.117

Yue, X.-Y., Li, X.-L., Wang, W.-W., Chen, D., Qiu, Q.-Q., Wang, Q.-C., et al. (2019). Wettable carbon felt framework for high loading Li-metal composite anode. Nano Energy 60, 257-266. doi: 10.1016/j.nanoen.2019.03.057 
Zhang, D., Zhou, Y., Liu, C., and Fan, S. (2016). The effect of the carbon nanotube buffer layer on the performance of a Li metal battery. Nanoscale 8, 11161-11167. doi: 10.1039/C6NR00465B

Zhang, H., Liao, X., Guan, Y., Xiang, Y., Li, M., Zhang, W., et al. (2018). Lithiophilic-lithiophobic gradient interfacial layer for a highly stable lithium metal anode. Nat. Commun. 9:3729. doi: 10.1038/s41467-018-06126-Z

Zhang, H., Xia, Y., Bu, H.,Wang, X., Zhang,M., Luo, Y., et al. (2013). Graphdiyne: a promising anode material for lithiumion batteries with high capacity and rate capability. J. Appl. Phys. 113:44309. doi: 10.1063/1.4789635

Zhang, H., Zhao, M., He, X., Wang, Z., Zhang, X., and Liu, X. (2011). High mobility and high storage capacity of lithium in sp-sp2 hybridized carbon network: the case of graphyne. J. Phys. Chem. C 115, 8845-8850. doi: 10.1021/jp201062m

Zhang, R., Chen, X. R., Chen, X., Cheng, X. B., Zhang, X. Q., Yan, C., et al. (2017). Lithiophilic sites in doped graphene guide uniform lithium nucleation for dendrite-free lithium metal anodes. Angew. Chem. 129, 7764-7768. doi: 10.1002/anie.201702099

Zhang, R., Cheng, X. B., Zhao, C. Z., Peng, H. J., Shi, J. L., Huang, J. Q., et al. (2016). Conductive nanostructured scaffolds render low local current density to inhibit lithium dendrite growth. Adv. Mater. 28, 2155-2162. doi: 10.1002/adma.201504117

Zhang, R., Wen, S., Wang, N., Qin, K., Liu, E., Shi, C., et al. (2018). NDoped graphene modified 3D porous $\mathrm{Cu}$ current collector toward microscale homogeneous Li deposition for Li metal anodes. Adv. Energy Mater. 8:1800914. doi: 10.1002/aenm.201800914

Zhang, S., Du, H., He, J., Huang, C., Liu, H., Cui, G., et al. (2016). Nitrogendoped graphdiyne applied for lithium-ion storage. ACS Appl. Mater. Interfaces 8, 8467-8473. doi: 10.1021/acsami.6b00255

Zhang, S., Liu, H., Huang, C., Cui, G., and Li, Y. (2015). Bulk graphdiyne powder applied for highly efficient lithium storage. Chem. Commun. 51, 1834-1837. doi: $10.1039 / \mathrm{C} 4 \mathrm{CC} 08706 \mathrm{~B}$
Zhao, Y., Sun, Q., Li, X., Wang, C., Sun, Y., Adair, K. R., et al. (2018). Carbon paper interlayers: a universal and effective approach for highly stable Li metal anodes. Nano Energy 43, 368-375. doi: 10.1016/j.nanoen.2017. 11.032

Zheng, G., Lee, S. W., Liang, Z., Lee, H. W., Yan, K., Yao, H., et al. (2014). Interconnected hollow carbon nanospheres for stable lithium metal anodes. Nat. Nanotechnol. 9, 618-623. doi: 10.1038/nnano. 2014.152

Zhi, W. S., Sun, Y., Zhang, Q., and Cui, Y. (2016). Designing high-energy lithium-sulfur batteries. Chem. Soc. Rev. 45, 5605-5634. doi: 10.1039/C5CS0 0410A

Zou, P., Wang, Y., Chiang, S. W., Wang, X., Kang, F., and Yang, C. (2018). Directing lateral growth of lithium dendrites in micro-compartmented anode arrays for safe lithium metal batteries. Nat. Commun. 9:464. doi: 10.1038/s41467-018-02888-8

Zuo, Z., and Li, Y. (2019). Emerging electrochemical energy applications of graphdiyne. Joule 3, 899-903. doi: 10.1016/j.joule.2019. 01.016

Conflict of Interest: The authors declare that the research was conducted in the absence of any commercial or financial relationships that could be construed as a potential conflict of interest.

Copyright (c) 2019 Liu, Li, Fan, Li, Maleki Kheimeh Sari and Qin. This is an openaccess article distributed under the terms of the Creative Commons Attribution License (CC BY). The use, distribution or reproduction in other forums is permitted, provided the original author(s) and the copyright owner(s) are credited and that the original publication in this journal is cited, in accordance with accepted academic practice. No use, distribution or reproduction is permitted which does not comply with these terms. 